ULB-TH/04-17

hep-th/0406192

final version

\title{
Parent field theory and unfolding in BRST first-quantized terms
}

\author{
G. Barnich, ${ }^{*, a}$ M. Grigoriev, ${ }^{\dagger, a, b}$ A. Semikhatov, ${ }^{b}$ and I. Tipunin ${ }^{b}$ \\ ${ }^{a}$ Physique Théorique et Mathématique and International Solvay Institutes, Uni- \\ versité Libre de Bruxelles, Campus Plaine C.P. 231, B-1050 Bruxelles, Bel- \\ gium \\ ${ }^{b}$ Tamm Theory Department, Lebedev Physics Institute, Leninsky prospect 53, \\ 119991 Moscow, Russia
}

\begin{abstract}
For free-field theories associated with BRST first-quantized gauge systems, we identify generalized auxiliary fields and pure gauge variables already at the first-quantized level as the fields associated with algebraically contractible pairs for the BRST operator. Locality of the field theory is taken into account by separating the space-time degrees of freedom from the internal ones. A standard extension of the first-quantized system, originally developed to study quantization on curved manifolds, is used here for the construction of a firstorder parent field theory that has a remarkable property: by elimination of generalized auxiliary fields, it can be reduced both to the field theory corresponding to the original system and to its unfolded formulation. As an application, we consider the free higher-spin gauge theories of Fronsdal.
\end{abstract}

\footnotetext{
* Senior Research Associate of the National Fund for Scientific Research (Belgium).

$\dagger$ Postdoctoral Visitor of the National Fund for Scientific Research (Belgium).
} 
1. Introduction 2

2. Local field theory associated with a BRST first-quantized system 4

2.1. BRST first-quantized system 4

$\begin{array}{lll}\text { 2.2. Local field theory and master action } & 6\end{array}$

2.3. Generalized auxiliary fields in the Lagrangian context 9

$\begin{array}{ll}3 . \quad \text { Non-Lagrangian BRST field theory } & 10\end{array}$

3.1. Equations of motion and gauge symmetries from a BRST differential 10

$\begin{array}{lll}\text { 3.2. Generalized auxiliary fields in the non-Lagrangian context } & 11\end{array}$

3.3. Elimination of generalized auxiliary fields at the first-quantized level 12

\begin{tabular}{|ll} 
3.4. The action for an equivalence class of equations of motion & 16
\end{tabular}

\begin{tabular}{lll}
\hline 3.5. First-quantized description of the Fronsdal Lagrangian & 17
\end{tabular}

$\begin{array}{lll}\text { 4. The first-order parent system } & 21\end{array}$

4.1. Extended first-quantized system and parent field theory 21

4.2. Reduction to the original system 23

4.3. Reduction to the unfolded formalism 25

4.4. From the parent theory to the unfolded formulation. Examples 26

5. Discussion 32

Appendix A. Structure of some polynomial sp(4) representations 34

\begin{tabular}{lll}
\hline Appendix B. Projectors & 37
\end{tabular}

$\begin{array}{ll}\text { References } & 38\end{array}$

\section{INTRODUCTION}

In the context of higher-spin theories, auxiliary fields have been prominent ever since they have been used by Fierz and Pauli [1] for the construction of Lagrangians for spin-2 and $3 / 2$ fields. This analysis has been completed in the case of arbitrary spin by Singh and Hagen [2, 3]. The massless limit of these generalized Lagrangians and the emergence of gauge invariance has then been studied by Fronsdal [4] and by Fang and Fronsdal [5].

Inspired by string field theory, the Fronsdal Lagrangians, with additional auxiliary fields, have been reproduced in the mid-eighties [6, 7, 8] as the Lagrangians associated with a first-quantized BRST system consisting of a truncation of the tensionless bosonic string. This reformulation turns out to be extremely convenient for constructing consistent 
interactions, extending to curved backgrounds or studying more general representations of the Lorentz group (see, e.g., [9, 10, 11, 12, 13, 14, 15, 16, 17]).

A different framework for higher-spin gauge theories known as the unfolded formalism was developed by M. Vasiliev [18, 19, 20] (see also [21, 22, 23] for a review and further developments). So far, only in this approach results on consistent interactions have been obtained to all orders. The unfolded formalism is explicitly space-time covariant and makes both the gauge and global symmetries of the theory transparent. This is because the free equations of motion have the form of a covariant constancy condition, which is achieved at the price of introducing an infinite tower of auxiliary fields. In this formulation, however, the equations do not seem to be naturally Lagrangian without the elimination or introduction of further auxiliary fields [24].

The aim of this paper is to explicitly relate the BRST-based approach and Vasiliev's method of unfolding in the case of free field theories. For this, we provide a detailed understanding, at the first-quantized level, of auxiliary fields, pure gauge degrees of freedom, and the issue of Lagrangian versus non-Lagrangian equations of motion. We construct an extended first-order BRST system whose associated field theory serves as a parent field theory: by elimination of auxiliary fields and pure gauge degrees of freedom, it can be reduced either to the starting point theory or to its unfolded formulation.

More precisely, we first recall in Sec. 2 how to associate a gauge field theory with a BRST first-quantized system along the lines developed in the context of string field theory. Technically, the BRST operator of the first-quantized system determines both the Batalin-Vilkovisky master action and the BRST differential of the gauge field theory. Special care is devoted to locality by treating the space-time degrees of freedom $x^{\mu}, p_{\nu}$ separately from internal degrees of freedom. We also recall that working on the level of the master action including ghosts and antifields allows giving a unified description of standard auxiliary fields and pure gauge degrees of freedom in terms of so-called generalized auxiliary fields.

Section 3 begins with the rather obvious remark that for a BRST first-quantized system, equations of motions, gauge symmetries, and the BRST differential of an associated field theory can be defined independently of the question of the existence of a Lagrangian. More importantly, for a possibly nonlinear field theory defined by some differential, we extend the definition of generalized auxiliary fields to this non-Lagrangian context.

For linear field theories associated with BRST systems, we then characterize generalized auxiliary fields at the first-quantized level as the fields associated with contractible pairs that can be eliminated algebraically in the computation of the BRST cohomology. In the same spirit, existence of a Lagrangian is understood in first-quantized terms as the existence of an inner product that makes the BRST operator formally self-adjoint. These 
general ideas are then illustrated by recalling explicitly how the Fronsdal Lagrangians can be obtained from a first-quantized BRST system.

In Sec. 4, we show how to make a given BRST system first-order in space-time derivatives via a procedure that is familiar from the problem of quantizing curved manifolds. The procedure implies the introduction of new internal degrees of freedom and an extended BRST operator such that the extended system is equivalent to the given BRST system. The extended BRST operator contains a space-time part and an internal part. Space-time derivatives enter only the space-time part, whereby the constraints describing the embedding of the original phase space into the extended one are taken into account. The internal part of the extended BRST operator coincides with the original BRST operator with space-time derivatives replaced by derivatives acting in the internal space enlarged by additional $y$ variables.

We then show that the extended system can be reduced to the original system by elimination of contractible pairs for the space-time part of the BRST differential. Another reduction consists in eliminating contractible pairs for the internal part of the BRST operator. The field theory of this latter reduction is related to the unfolded form of the equations of motion for the starting point field theory. Therefore, the field theory of the extended system can be regarded as a parent theory from which both the original and the unfolded formulations can be obtained via elimination of generalized auxiliary fields. We illustrate the formalism in the case of the Klein-Gordon equation and the free gauge theories for fields of integer higher spins.

Finally, we briefly comment on the validity of our analysis in the case of nonflat backgrounds and discuss symmetries and interactions. The appendix is devoted to the cohomology of the internal part of the extended BRST operator for the Fronsdal system.

\section{LOCAL FIELD THEORY ASSOCIATED WITH A BRST FIRST-QUANTIZED SYSTEM}

In this section, we recall some elements of the standard operator BRST formalism, which we use to describe first-quantized systems of the type "particle with internal degrees of freedom." Examples of such systems include the spinless relativistic particle, higher-spin particle models [6, 7, 8], and strings. In Sec. 2.2 we then consider a gauge field theory whose fields are associated with the wave functions of the first-quantized system. A Lagrangian for this field theory is constructed using the BRST operator whenever an inner product exists on the space of first-quantized wave functions. In Sec. 2.3 we recall the concept of generalized auxiliary fields whose introduction/elimination provides one with a natural notion of equivalence for such gauge field theories.

2.1. BRST first-quantized system. We consider a constrained Hamiltonian system with phase space $T^{*} \mathcal{X} \times B$, where $T^{*} \mathcal{X}$ is the cotangent bundle to a manifold $\mathcal{X}$ and $B$ is a 
symplectic supermanifold. The constraints are given by

$$
f_{\alpha}=0, \quad \alpha=1, \ldots, s,
$$

where $f_{\alpha}$ are functions on $T^{*} \mathcal{X} \times B$. This system can be considered as a particle on $X$ with internal degrees of freedom described by $B$.

In the BRST approach to constrained dynamics [25, 26, 27] (see also [28]), a ghost $c^{\alpha}$ and the corresponding momentum $\mathcal{P}_{\alpha}$ must be introduced for each constraint $f_{\alpha}$. The Grassmann parities of both $c^{\alpha}$ and $\mathcal{P}_{\alpha}$ are opposite to those of $f_{\alpha}$. Let $\Lambda$ be the linear supermanifold with coordinates $c^{\alpha}$. The extended phase space of the BRST system is then $T^{*} X \times B \times T^{*} \Lambda$, with the obvious symplectic structure. The ghost-number grading is introduced on functions on $T^{*} \mathcal{X} \times B \times T^{*} \Lambda$ such that $\operatorname{gh}\left(c^{\alpha}\right)=1, \operatorname{gh}\left(\mathcal{P}_{\alpha}\right)=-1$.

Given the constraints, one constructs the classical BRST charge $\Omega^{c l}$ on the extended phase space, which is a Grassmann-odd and ghost-number-one function satisfying

$$
\left\{\Omega^{c l}, \Omega^{c l}\right\}=0, \quad \Omega^{c l}=c^{\alpha} f_{\alpha}+\text { more. }
$$

The differential $\left\{\Omega^{c l}, \cdot\right\}$ provides a resolution of the algebra of functions on the reduced phase space. Inequivalent physical observables then correspond to ghost-number-zero elements of the cohomology space of $\left\{\Omega^{c l}, \cdot\right\}$ evaluated in the algebra of functions on the extended phase space. The Poisson structure on the extended phase space naturally induces a Poisson bracket in cohomology. We consider two classical BRST systems equivalent if their ghost-number-zero cohomology spaces are isomorphic as Poisson algebras.

We next consider the quantization of this system assuming that $\mathcal{X}$ is $\mathbb{R}^{d}$. The states are taken to be sections of the bundle

$$
\mathcal{H}=X \times \mathcal{H} \rightarrow X
$$

where $\mathcal{H}$ is the vector (super)space of states arising in the quantization of $B \times T^{*} \Lambda$, which we assume to be equipped with a sesquilinear inner product $\langle\cdot, \cdot\rangle_{\mathcal{H}}$. The quantum operator algebra inherits the Grassmann parity and ghost number from the classical level, but in the representation space $\mathcal{H}$, both gradings are defined modulo suspension by a constant. ${ }^{1}$ The following properties of the sesquilinear inner product are assumed:

$$
\overline{\langle\phi, \psi\rangle_{\mathcal{H}}}=(-1)^{|\phi||\psi|}\langle\psi, \phi\rangle_{\mathcal{H}}, \quad\langle\phi \alpha, \psi \beta\rangle_{\mathcal{H}}=\langle\phi, \psi\rangle_{\mathcal{H}} \bar{\alpha} \beta, \quad \alpha, \beta \subset \mathbb{C},
$$

where $|\cdot|$ denotes Grassmann parity. We also assume that an additional grading in $\mathcal{H}$ can be introduced such that each graded component is finite-dimensional.

In terms of a real basis $\left(e_{A}\right)$ in $\mathcal{H}$, the sections of $\mathcal{H}$ are written as

$$
\phi(x)=e_{A} \phi^{A}(x),
$$

\footnotetext{
${ }^{1}$ For a system without physical fermions, the two gradings are compatible in the sense that parity corresponds to ghost number modulo 2 .
} 
where $\left(x^{\mu}\right), \mu=1, \ldots, d$, are coordinates on $X$ and the convention of summation over repeated indices is understood. If $\left(p_{\mu}\right)$ are coordinates on the fibers of the cotangent bundle $T^{*} \mathcal{X}$, the operators $x^{\mu}$ and $p_{\mu}$ and their commutation relations $\left[p_{\nu}, x^{\mu}\right]=-\imath \delta_{\nu}^{\mu}$ are represented on sections $\Gamma(\mathcal{H})$ of $\mathcal{H}$ in the standard way through multiplication and derivation with $x^{\mu}$.

We assume that under quantization, the BRST charge $\Omega^{c l}$ becomes a nilpotent Hermitian operator $\Omega$ on $\Gamma(\mathcal{H})$. We additionally require $\Omega$ to contain a finite number of derivatives with respect to $x$. The first-quantized system $(\Omega, \Gamma(\mathcal{H}))$ is thus determined by the BRST operator $\Omega$ and the space of states $\Gamma(\mathcal{H})$.

2.2. Local field theory and master action. We now associate a local field theory with the first-quantized system $(\Omega, \Gamma(\mathcal{H}))$. Given a real basis $\left(e_{A}\right)$ in $\mathcal{H}$, we consider the dual basis $\left(\psi^{A}\right)$, with the assignments $\operatorname{gh}\left(\psi^{A}\right)=-\operatorname{gh}\left(e_{A}\right)$ and with the Grassmann parities $\left|\psi^{A}\right|=\left|e_{A}\right|$, and let $\mathcal{A}_{\mathcal{H}}$ be the algebra of polynomials in the $\left(\psi^{A}\right)$. We then consider $\left(\psi^{A}\right)$ as coordinates on a supermanifold $\mathcal{M}_{\mathcal{H}}$ (that is, $\mathcal{M}_{\mathcal{H}}$ is the affine supermanifold whose algebra of functions is the associative supercommutative algebra $\mathcal{A}_{\mathcal{H}}$ ). We view $\mathcal{M}_{\mathcal{H}}$ as a real supermanifold. The inner product $\langle\cdot, \cdot\rangle_{\mathcal{H}}$ on $\mathcal{H}$ then determines two forms on $\mathcal{M}_{\mathcal{H}}$, a symmetric and a symplectic one, in accordance with (see [29, 30] and also [31, 32, 33, 34] $)^{2}$

$$
\begin{gathered}
\langle\psi, \phi\rangle_{\mathcal{H}}=g(\psi, \phi)+i \omega(\psi, \phi), \\
g_{A B}=(-1)^{(\kappa+1)\left|e_{A}\right|} g\left(e_{A}, e_{B}\right), \quad \omega_{A B}=(-1)^{(\kappa+1)\left|e_{A}\right|} \omega\left(e_{A}, e_{B}\right),
\end{gathered}
$$

where we use $\kappa$ to denote the Grassmann parity of $\langle\cdot, \cdot\rangle_{\mathcal{H}}$ and hence of the forms $g$ and $\omega$. In what follows, we only consider the case where the inner product is odd and of the ghost number -1 .

We assume that $\mathcal{H}$ is decomposed with respect to the ghost number,

$$
\mathcal{H}=\bigoplus_{k} \mathcal{H}^{(k)}
$$

where elements of $\mathcal{H}^{(k)}$ are of ghost number $-k$. If $e_{A_{k}}$ denotes basis elements in $\mathcal{H}^{(k)}$, then $\operatorname{gh}\left(\psi^{A_{k}}\right)=k=-\operatorname{gh}\left(e_{A_{k}}\right)$.

We next take the space of fields to be the space of (suitably smooth) maps from $X$ to the supermanifold $\mathcal{M}_{\mathcal{H}}$. The fields $\psi^{A}(x)$ then inherit the ghost number and Grassmann parity. In particular, $\psi^{A_{0}}(x)$ denote fields associated with basis elements in the zeroghost-number subspace $\mathcal{H}^{(0)}$. These are interpreted as physical fields; the remaining fields are identified with auxiliary BRST variables (ghost fields and antifields).

\footnotetext{
${ }^{2}$ In the string field theory literature, a complex bilinear version of the symplectic structure is considered as a starting point and is related to the sesquilinear inner product by an appropriate antilinear involution (see, e.g., [35]). On the associated real form of $\mathcal{H}$, both these symplectic structures coincide.
} 
We define the action for the physical fields as

$$
\boldsymbol{S}^{\mathrm{ph}}[\psi]=-\frac{1}{2} \int d^{d} x g_{A_{0} B_{-1}} \psi^{A_{0}} \Omega_{C_{0}}^{B_{-1}} \psi^{C_{0}}
$$

where the differential operators $\Omega_{C_{0}}^{B-1}$ are defined as

$$
\Omega \phi=\Omega\left(e_{A_{0}} \phi^{A_{0}}(x)\right)=e_{B_{-1}} \Omega_{A_{0}}^{B_{-1}} \phi^{A_{0}}(x), \quad \phi \in \Gamma\left(\mathcal{H}^{(0)}\right)
$$

and $\Gamma\left(\mathcal{H}^{(0)}\right)$ is the space of zero-ghost-number sections of $\mathcal{H}$. The Euler-Lagrange equations of motion following from $S^{\mathrm{ph}}$ are given by

$$
\Omega_{B_{0}}^{A_{-1}} \psi^{B_{0}}(x)=0 .
$$

Because $\Omega^{2}=0$, the action (2.8) possesses the gauge symmetries $\delta_{\epsilon} \psi^{A_{0}}=\Omega^{A_{0}}{ }_{B_{1}} \epsilon^{B_{1}}$, for some gauge parameters $\epsilon^{B_{1}}$. For the same reason, these gauge symmetries are in general reducible.

Before giving the Batalin-Vilkovisky master action associated with this gauge theory, we introduce the concept of string field in the context of local field theory. Let $\mathcal{A}$ be the algebra of local functions associated with the fields $\psi^{A}(x)$, i.e., the algebra of functions depending on $x^{\mu}, \psi^{A}$, and $\psi_{\mu_{1}}^{A}, \psi_{\mu_{1} \mu_{2}}^{A}, \ldots, \psi_{\mu_{1} \ldots \mu_{n}}^{A}$ up to some finite order (see, e.g., [36], [37). The ghost-number assignments and the Grassmann parities of $\psi_{\mu_{1} \ldots \mu_{n}}^{A}$ coincide with those of $\psi^{A}$. The space of local functionals $\mathcal{F}_{\mathcal{A}}$ is the quotient of the space of local functions modulo those of the form $\partial_{\mu} j^{\mu}$, where $\partial_{\mu}$ denotes the total derivative with respect to $x^{\mu}$, i.e., the vector field

$$
\partial_{\mu}=\frac{\partial}{\partial x^{\mu}}+\psi_{, \mu}^{A} \frac{\partial}{\partial \psi^{A}}+\psi_{, \mu \nu}^{A} \frac{\partial}{\partial \psi_{, \nu}^{A}}+\ldots
$$

We use the integral sign $\int d^{d} x$ to denote projection from local functions to local functionals.

We consider the right $\mathcal{A}$-module $\mathcal{H} \otimes \mathcal{A}$. The sesquilinear form on $\mathcal{H}$ is naturally extended to $\mathcal{H} \otimes \mathcal{A}$ as

$$
\langle\psi \otimes \boldsymbol{f}, \phi \otimes \boldsymbol{g}\rangle=(-1)^{|\boldsymbol{f}||\boldsymbol{\phi}|}\langle\psi, \phi\rangle_{\mathcal{H}} \boldsymbol{f} \boldsymbol{g}, \quad \boldsymbol{f}, \boldsymbol{g} \in \mathcal{A},
$$

and the forms $g(\cdot, \cdot)$ and $\omega(\cdot, \cdot)$ (see (2.6) ) are extended similarly. Applying the projection $\int d^{d} x$ to (2.12) gives a bilinear form on $\mathcal{H} \otimes \mathcal{A}$ with values in local functionals.

The odd symplectic structure on $\mathcal{M}_{\mathcal{H}}$ induces a map $(\cdot, \cdot): \mathcal{F}_{\mathcal{A}} \otimes \mathcal{A} \longrightarrow \mathcal{A}$ defined by

$$
(\boldsymbol{F}, \boldsymbol{g})=\frac{\delta^{R} \boldsymbol{f}}{\delta \psi^{A}} \omega^{A B} \frac{\partial^{L} \boldsymbol{g}}{\partial \psi^{B}}+\partial_{\mu}\left(\frac{\delta^{R} \boldsymbol{f}}{\delta \psi^{A}} \omega^{A B}\right) \frac{\partial^{L} \boldsymbol{g}}{\partial \psi_{, \mu}^{B}}+\ldots,
$$

where $\boldsymbol{F}=\int d^{d} x \boldsymbol{f} \in \mathcal{F}_{\mathcal{A}}$ and $\boldsymbol{g} \in \mathcal{A}$ with $\frac{\delta}{\delta \psi^{A}}$ denoting the Euler-Lagrange derivative with respect to $\psi^{A}$. We note that the vector field $(\boldsymbol{F}, \cdot)$ commutes with $\partial_{\mu}$, which implies that there is a well-defined bracket induced in the space of local functionals. This bracket can be shown to be an odd graded Lie bracket, called antibracket in what follows. 
Linear operators acting on $\Gamma(\mathcal{H})$ are also extended to $\mathcal{H} \otimes \mathcal{A}$. Namely, identifying the space of linear operators on $\Gamma(\mathcal{H})$ with $\operatorname{Diff}(\mathcal{X}) \otimes \operatorname{End}(\mathcal{H})$, where $\operatorname{Diff}(\mathcal{X})$ is the space of differential operators on $\mathcal{X}$ (and $\operatorname{End}(\mathcal{H})$ is the space of linear operators on $\mathcal{H}$ ), we define

$$
(A \otimes O)(\phi \otimes \boldsymbol{f})=(A \phi) \otimes(O \boldsymbol{f}), \quad A \in \operatorname{End}(\mathcal{H}), O \in \operatorname{Diff}(X), \phi \in \mathcal{H}, \boldsymbol{f} \in \mathcal{A},
$$

where the action of $O$ on $\boldsymbol{f} \in \mathcal{A}$ is defined by replacing each $x^{\mu}$-derivative with $\partial_{\mu}$ given in (2.11).

The string field $\Psi$ is the element of the module $\mathcal{H} \otimes \mathcal{A}$ defined as

$$
\Psi=e_{A} \otimes \psi^{A} \text {. }
$$

We omit $\otimes$ in what follows. We often refer to $\Psi$ as the string field associated with $\mathcal{H}$. For any subspace $\mathcal{V} \subset \mathcal{H}$, there is a restriction of $\Psi$ to a string field associated with $\mathcal{V}$. In particular, taking $\mathcal{V}$ to range over the subspaces $\mathcal{H}^{(k)}$ in (2.7) gives the decomposition $\Psi=\sum_{k} \Psi^{(k)}$ with $\Psi^{(k)}=e_{A_{k}} \psi^{A_{k}}=\left.\Psi\right|_{\mathcal{H}^{(k)}}$; the components of $\Psi^{(0)}$ are physical fields $\psi^{A_{0}}$. In terms of the string field, expression (2.8) takes the form

$$
\boldsymbol{S}^{\mathrm{ph}}\left[\Psi^{(0)}\right]=-\frac{1}{2} \int d^{d} x\left\langle\Psi^{(0)}, \Omega \Psi^{(0)}\right\rangle .
$$

The local functional

$$
\boldsymbol{S}[\Psi]=-\frac{1}{2} \int d^{d} x\langle\Psi, \Omega \Psi\rangle,
$$

satisfies the master equation $(\boldsymbol{S}, \boldsymbol{S})=0$ and $\left.\boldsymbol{S}[\Psi]\right|_{\Psi^{(l)=0, l \neq 0}}=\boldsymbol{S}^{p h}\left[\Psi^{(0)}\right]$ and is therefore the master action [38, 39, 40, 41] associated with (2.16). We then consider the BRST differential

$$
s=(S, \cdot) .
$$

The cohomology of this differential in the space of local functionals contains information on consistent interactions and on (generalized) global symmetries [42, 43, 44].

\section{Remarks.}

(i) Alternatively, one can think of $\Gamma(\mathcal{H})$ as a Hilbert space with basis vectors $e_{A}^{x}=$ $|x\rangle \otimes e_{A}$. Instead of (2.15), one then obtains

$$
\Psi=\int d x|x\rangle \otimes e_{A} \psi^{A}(x)
$$

as the string field. We prefer using $\Psi$ defined in (2.15) in order to avoid additional assumptions needed to work with (2.19).

(ii) In constructing the field theory, we used real fields $\psi^{A}$ associated with a real basis $\left(e_{A}\right)$ in $\mathcal{H}$. Equivalently, one can use complex fields associated with a complex basis in $\mathcal{H}$ (see, e.g., [35, 34] for more details). This option is used in Secs. 3.5 and 4.4 
2.3. Generalized auxiliary fields in the Lagrangian context. For a Lagrangian field theory determined by $\boldsymbol{S}^{\mathrm{ph}}$, ordinary auxiliary fields are fields $v^{a}$ whose Euler-Lagrange equations $\frac{\delta \boldsymbol{S}^{\mathrm{ph}}}{\delta v^{a}}=0$ can be solved algebraically for $v^{a}$. In this subsection we recall the definition of generalized auxiliary fields in the Lagrangian context [45].

For a given action $\boldsymbol{S}[\psi]$ that depends on some variables $\psi^{A}$ and satisfies the master equation $(\boldsymbol{S}, \boldsymbol{S})=0$, we suppose that the $\psi^{A}$ can be split into $w^{a}, w_{a}^{*}, \varphi^{\gamma}$ such that the equations

$$
\frac{\delta \boldsymbol{S}}{\delta w^{a}}=0
$$

can be solved algebraically for $w^{a}$ at $w_{a}^{*}=0$. Let then $w^{a}=W^{a}[\varphi]$ be the solution, i.e.,

$$
\left.\frac{\delta \boldsymbol{S}}{\delta w^{a}}\right|_{\Sigma} \equiv 0
$$

where $\Sigma$ is determined by the constraints $w^{a}-W^{a}[\varphi]=0, w_{a}^{*}=0$ and their space-time derivatives. If $w^{a}$ and $w_{a}^{*}$ are canonically conjugate with respect to the antibracket (and therefore the defining relations for $\Sigma$ are second-class constraints), we say that $w^{a}, w_{a}^{*}$ are generalized auxiliary fields.

Generalized auxiliary fields can be consistently eliminated in the sense that the master action pulled back to $\Sigma$ satisfies the master equation with respect to the corresponding Dirac antibracket. Elimination of generalized auxiliary fields is a natural equivalence of local gauge theories, in particular, (local) BRST cohomology groups are invariant under the elimination of generalized auxiliary fields. In addition to the elimination of ordinary auxiliary fields and their conjugate antifields, the elimination of generalized auxiliary fields includes the elimination of pure (algebraic) gauge degrees of freedom together with their associated ghosts and antifields.

We now give an example of generalized auxiliary fields which can already be identified at the first-quantized level (see [34] for more details). Consider the situation where $\mathcal{H}$ has the form $\mathcal{H}=\mathcal{U} \otimes \mathcal{V}$ and where the inner product on $\mathcal{H}$ is the tensor product of an inner product on $\mathcal{U}$ and an inner product $\langle\cdot, \cdot\rangle_{\mathcal{V}}$ on $\mathcal{V}$. Suppose that the BRST differential on $\Gamma(\mathcal{H}) \cong \Gamma(\mathcal{U}) \otimes \mathcal{V}$ is of the form $\Omega=\Omega_{\mathcal{U}} \otimes \mathbf{1}+\mathbf{1} \otimes \Omega^{\prime}$, where $\Omega^{\prime}$ is a nilpotent Hermitian operator on $\mathcal{V}$ with respect to $\langle\cdot, \cdot\rangle_{\mathcal{V}}$. Suppose furthermore that $\mathcal{V}$ decomposes into a singlet and null doublets as well as quartets with respect to $\Omega^{\prime}$ and $\langle\cdot, \cdot\rangle_{\mathcal{V}}$ (see, e.g., [46] for details on irreducible representations of BRST algebra). Then the fields carrying the index associated with the null doublets are ordinary auxiliary fields and their associated antifields, while those carrying the index of quartets are generalized auxiliary fields. After their elimination, we obtain an action of the form (2.17) associated with $\mathcal{U}$ alone. 


\section{NON-LAGRANGIAN BRST FIELD THEORY}

In the previous section, we constructed a particular Lagrangian gauge field theory. We now show that some basic features of the construction can be extended to the nonLagrangian context. For a BRST first-quantized system, we just take over the previous definitions of equations of motion and of gauge symmetries. For possibly nonlinear gauge field theories defined by a differential $s$, we define generalized auxiliary fields in terms of the differential $s$ and show that their elimination or introduction does not affect various cohomologies of $s$. We then go back to the not necessarily Lagrangian field theory defined by a BRST first-quantized system and study the first-quantized counterpart of generalized auxiliary fields and their elimination. We show that the existence of a Lagrangian for the field theory associated with a BRST first-quantized system is equivalent to the existence of an inner product that makes the BRST operator formally self-adjoint. Finally, we illustrate these concepts in the case of the Fronsdal system.

3.1. Equations of motion and gauge symmetries from a BRST differential. In the case where an inner product does not exist on $\mathcal{H}$ (or is not specified), one can still consider the equations of motion (2.10) for the physical fields $\psi^{A_{0}}, \operatorname{gh}\left(\psi^{A_{0}}\right)=0$ contained in $\Psi^{(0)}$. These equations are then not necessarily equivalent to variational ones. In this context, one can also define the BRST differential

$$
s=(\Omega \Psi)^{A} \frac{\partial}{\partial \psi^{A}}+\partial_{\mu}\left[(\Omega \Psi)^{A}\right] \frac{\partial}{\partial \psi_{, \mu}^{A}}+\ldots,
$$

satisfying $\left[s, \partial_{\mu}\right]=0$ where $\partial_{\mu}$ is defined in 2.11. The BRST differential determines the equations of motion through

$$
s \Psi^{(-1)} \equiv \Omega \Psi^{(0)}=0
$$

The differential $s$ in (3.1) is linear in $\Psi$. More generally, we consider a not necessarily linear differential (still denoted by $s$ and called the BRST differential) acting on a space of local functions. We write $\psi^{A}(x)$ for the components of these functions with respect to a basis $\left(e_{A}\right)$, with $\Psi(x)=e_{A} \psi^{A}(x)$. As before, we also assume that the space of fields is graded by ghost number, see (2.7), with $\Psi^{(\ell)}$ being the string field associated with $\mathcal{H}^{(\ell)}$. The BRST differential $s$ is considered to have ghost number 1 . This differential determines equations of motion for the physical fields as

$$
\left.s \Psi^{(-1)}\right|_{\Psi^{(\ell)}=0, \ell \neq 0}=0,
$$

By expanding $s^{2}=0$ according to the ghost number, it can be shown that the transformations

$$
\delta \Psi^{(0)}=\left.s \Psi^{(0)}\right|_{\Psi^{(\ell)}=0, \ell \neq 0,1}
$$


with ghost-number-1 component fields of $\Psi^{(1)}$ replaced by gauge parameters, are gauge symmetries of Eqs. (3.3). ${ }^{3}$

3.2. Generalized auxiliary fields in the non-Lagrangian context. Generalized auxiliary fields, defined in $\mathbf{2 . 3}$ in the Lagrangian context, can also be defined for a nonLagrangian system described by some differential $s$ that is not necessarily linear in $\psi^{A}$.

Let $\mathcal{V}$ be an even-dimensional subspace in $\mathcal{H}$ and let $\left(e_{\alpha}, f_{a}, g_{a}\right)$, be a basis in $\mathcal{H}$ such that $\left(f_{a}, g_{a}\right)$ form a basis in $\mathcal{V}$. Let then $\varphi^{\alpha}, v^{a}, w^{a}$ be the fields associated with the respective basis elements $e_{\alpha}, f_{a}, g_{a}$. We also suppose that the equations

$$
\left.\left(s w^{a}\right)\right|_{w^{a}=0}=0
$$

can be solved algebraically for $v^{a}$ as $v^{a}=V^{a}[\varphi]$. In this context, we still refer to the fields $v^{a}$ and $w^{a}$ as generalized auxiliary fields.

The differential $s$ then naturally restricts to a differential $\widetilde{s}$ on the surface $\Sigma$ defined by

$$
w^{a}=0, \quad v^{a}-V^{a}[\varphi]=0,
$$

and their space-time derivatives. Indeed, $s$ preserves the ideal of local functions vanishing on $\Sigma$ : because this ideal is generated by $w^{a}, v^{a}-V^{a}[\varphi]$, and their derivatives with respect to coordinates $x^{\mu}$ on the base manifold, it suffices to show that $\left.s w^{a}\right|_{\Sigma}=0$ and $s\left(v^{a}-\right.$ $\left.V^{a}[\varphi]\right)\left.\right|_{\Sigma}=0$. The first equation is tautological, while the second is a consequence of the nilpotency of $s$ and the representation

$$
v^{a}-V^{a}[\varphi]=A_{b}^{a} s w^{b}+B_{b}^{a} w^{b},
$$

where $A_{b}^{a}$ and $B_{b}^{a}$ are differential operators and standard regularity conditions on Eqs. (3.6) have been assumed.

It follows from the definition that generalized auxiliary fields can be organized in subsets $v^{a_{k}}$ and $w^{a_{k-1}}$ of the respective ghost numbers $k$ and $k-1$

Proposition 3.1. Let $v^{a}$ and $w^{a}$ be generalized auxiliary fields. Let also $\operatorname{gh}\left(v^{a}\right)=k$ and $\operatorname{gh}\left(w^{a}\right)=k-1$.

(1) For $k \neq 0$, 1, Eqs. (3.3) are unchanged by elimination of $v, w$.

(2) For $k=0$, the fields $v^{a}$ are ordinary auxiliary fields in the sense that Eqs. (3.3) can be solved algebraically for $v^{a}$.

(3) For $k=1$, the fields $w^{a}$ are pure gauge in the sense that they are not constrained by Eqs. (3.3). Furthermore, arbitrary shifts in these variables can be generated by gauge transformations determined by sor an appropriate choice of gauge parameters.

\footnotetext{
${ }^{3}$ By gauge symmetries of equations of motion 3.3 , we understand transformations that depend on arbitrary parameters and that leave the ideal of local functions generated by the left-hand sides of 3.3 invariant.
} 
Proof. Assertion 1 follows because for $k, k-1 \neq 0$, the fields $v^{a}, w^{a}$ have nonvanishing ghost numbers, and therefore do not enter the equations of motion.

For $k=0$, the fields $v^{a}$ carry ghost number 0 and the equations $\left.s w^{a}\right|_{w=0}=0$ can be solved algebraically for $v^{a}$. This implies that the equations $\left.s w^{a}\right|_{\Psi^{(\ell)}=0, \ell \neq 0}=0$ can still be solved algebraically because no $v^{a}$ are set to zero by putting all $\Psi^{(l)}$ for $l \neq 0$ to zero. At the same time, these equations form a subset of Eqs. (3.3), and we conclude that $v^{a}$ are ordinary auxiliary fields.

To show assertion 3, we introduce new independent fields $w^{a}, \chi^{a}=s w^{a}$, and $\varphi^{\alpha}$. In these coordinates, $s$ takes the form

$$
s=Q^{\alpha} \frac{\partial}{\partial \varphi^{\alpha}}+\chi_{a} \frac{\partial}{\partial w^{a}}+\partial_{\mu} Q^{\alpha} \frac{\partial}{\partial \varphi_{, \mu}^{\alpha}}+\partial_{\mu} \chi_{a} \frac{\partial}{\partial w_{, \mu}^{a}}+\ldots,
$$

where $Q^{\alpha}=s \varphi^{\alpha}$, which implies the statement about shift symmetries. We can further modify this coordinate system by redefining $\varphi^{\alpha}$ to $\varphi^{1 \alpha}=\varphi^{\alpha}-\rho Q^{\alpha}$, where $\rho=w^{a} \frac{\partial}{\partial \chi^{a}}$. Then $s \varphi^{1 \alpha} \equiv Q^{1 \alpha}$ can be easily seen not to contain terms linear in $\chi^{a}$. From the consistency condition $s Q^{1 \alpha}=0$, it then follows that $Q^{1 \alpha}$ does not contain terms linear in $w^{a}$ either. Induction on homogeneity degree in both $\chi^{a}, w^{a}$ together with expansions in $\chi^{a}$ then shows, along similar lines, that the dependence on $\chi^{a}$ and $w^{a}$ can be completely absorbed through all orders by appropriate redefinitions of $\varphi^{\alpha}$. This implies that the equations of motion $\left.Q^{\alpha}\right|_{\Psi^{(\ell)}=0, \ell \neq 0}=0$ do not involve $w^{a}$ in these coordinates.

Given the BRST differential $s$, we can consider its cohomology groups in the space of local functions, in the space of local functionals, the cohomology of the adjoint action $[s, \cdot]$ in the space of evolutionary vector fields or the cohomology of $s$ modulo $\boldsymbol{d}=d x^{\mu} \partial_{\mu}$ in the space of horizontal forms. Here, evolutionary vector fields are those which commute with $\partial_{\mu}$ (see, e.g., [36]), horizontal forms are differential forms in $d x^{\mu}$ with coefficients in local functions, and $\partial_{\mu}$ is the total derivative. As a consequence of representation (3.8), we see that all the above cohomology groups are invariant under elimination of generalized auxiliary fields. Somewhat more formally, we summarize this as the following proposition.

Proposition 3.2. If $v^{a}$ and $w^{a}$ are generalized auxiliary fields for a differential $s$ and $\widetilde{s}$ is the restriction of $s$ to the surface $\Sigma$ defined by (3.6), then $s$ and $\widetilde{s}$ have the same cohomology.

3.3. Elimination of generalized auxiliary fields at the first-quantized level. We now concentrate on the case where the BRST differential $s$ originates from the first-quantized BRST operator $\Omega$ as in (3.1). At the first-quantized level, we can then identify the states that correspond to (generalized) auxiliary fields. Namely, as a first-quantized counterpart of the setting at the beginning of Sec. 3.2, we suppose that

$$
\mathcal{H}=\mathcal{E} \oplus \mathcal{F} \oplus \mathcal{G}, \quad \operatorname{dim} \mathcal{G}=\operatorname{dim} \mathcal{F}
$$


(where in the graded-finite-dimensional case, the dimensions of the corresponding graded components must be equal). Elements of $\Gamma(\mathcal{H})$ and the BRST operator can then be decomposed accordingly,

$$
\begin{aligned}
& \phi(x)=\phi^{\mathcal{E}}(x)+\phi^{\mathcal{F}}(x)+\phi^{\mathcal{G}}(x), \\
& \left.\stackrel{\bullet_{1} \bullet_{2}}{\Omega} \phi\right|_{\bullet_{2}}=\left.\left(\Omega\left(\left.\phi\right|_{\bullet_{2}}\right)\right)\right|_{\bullet_{1}},
\end{aligned}
$$

where $\bullet_{1}, \bullet_{2}$ run over $\mathcal{E}, \mathcal{F}, \mathcal{G}$. Of course, this decomposition extends to the string field: $\Psi=\Psi^{\mathcal{E}}+\Psi^{\mathcal{F}}+\Psi^{\mathcal{G}}$, where $\Psi^{\mathcal{X}}=\left.\Psi\right|_{\mathcal{X}}$ (see Sec. 2.2) for simplicity of notation.

We note that here and below, we consider differential operators with a finite (or graded finite) number of derivatives. If an operator is invertible in this space, we call it algebraically invertible.

Lemma 3.3. Given decomposition (3.9), the fields $v^{a}$ and $w^{a}$ associated with the respective basis elements $f_{a}$ and $g_{a}$ of $\mathcal{F}$ and $\mathcal{G}$ are generalized auxiliary fields if and only if $\Omega$ is algebraically invertible.

Proof. Indeed, the equations $\left.\left(s w^{a}\right)\right|_{w=0}=0$ become $\left.\left(\left.(\Omega \Psi)\right|_{\mathcal{G}}\right)\right|_{\Psi^{\mathcal{G}}=0}=0$, or equivalently,

$$
\stackrel{\mathcal{G F}}{\Omega \mathcal{F}} \Psi^{\mathcal{F}}+\stackrel{\mathcal{G} \mathcal{E}}{\Omega} \Psi^{\mathcal{E}}=0 .
$$

This equation can be solved algebraically for the component fields of $\Psi^{\mathcal{F}}$ in terms of the component fields of $\Psi^{\mathcal{E}}$ and a (graded) finite number of derivatives if and only if $\Omega$ $\Omega$ is invertible as a differential operator with the inverse containing a (graded) finite number of derivatives. Hence, $\mathcal{F} \oplus \mathcal{G} \subset \mathcal{H}$ corresponds to $\mathcal{V}$ in the definition of generalized auxiliary fields in 3.2.

The reduced differential $\widetilde{s}$ is determined by the BRST operator

$$
\widetilde{\Omega}=\left(\Omega \mathcal{E}\left(\mathcal{E F} \mathcal{G} \mathcal{G}{ }^{\mathcal{F}}\right)^{-1} \mathcal{G \mathcal { E }}\right): \Gamma(\mathcal{E}) \rightarrow \Gamma(\mathcal{E})
$$

such that

$$
\left.\widetilde{s} \Psi^{\mathcal{E}} \equiv(\Omega \Psi)^{\mathcal{E}}\right|_{\Psi^{\mathcal{G}}=0, \Psi^{\mathcal{F}}=\Psi^{\mathcal{F}}\left(\Psi^{\mathcal{E}}\right)}=\widetilde{\Omega} \Psi^{\mathcal{E}},
$$

where we write $\Psi^{\mathcal{F}}=\Psi^{\mathcal{F}}\left(\Psi^{\mathcal{E}}\right)$ for the solution of (3.11) for $\Psi^{\mathcal{F}}$. Hence,

Proposition 3.4. Let $\stackrel{\mathcal{G F}}{\Omega}$ be algebraically invertible. By elimination of the corresponding generalized auxiliary fields, the field theory associated with $(\Omega, \Gamma(\mathcal{H}))$ is reduced to the field theory associated with $(\widetilde{\Omega}, \Gamma(\mathcal{E}))$, where $\widetilde{\Omega}$ is defined in (3.12).

We call the reduction from the system $(\Omega, \Gamma(\mathcal{H}))$ to $(\widetilde{\Omega}, \Gamma(\mathcal{E}))$ algebraic because in the process of reduction, we stay in the space of differential operators with graded finite 
number of derivatives and because at the field theory level, this reduction corresponds to the algebraic elimination of generalized auxiliary fields.

Proposition 3.5. Let $\stackrel{\mathcal{G F}}{\Omega}$ be algebraically invertible and $\widetilde{\Omega}$ be defined by (3.12). The first-quantized systems $(\Omega, \Gamma(\mathcal{H}))$ and $(\widetilde{\Omega}, \Gamma(\mathcal{E}))$ are then equivalent in the sense that $H(\Omega, \Gamma(\mathcal{H})) \cong H(\widetilde{\Omega}, \Gamma(\mathcal{E}))$.

Proof. Indeed, as we see momentarily, the map

$$
\begin{aligned}
\mathfrak{f}: \Gamma(\mathcal{E}) & \rightarrow \Gamma(\mathcal{H}), \\
\phi^{\mathcal{E}}(x) & \mapsto \phi^{\mathcal{E}}(x)-(\stackrel{\mathcal{G F}}{\Omega})^{-1} \stackrel{\mathcal{G E}}{\Omega} \phi^{\mathcal{E}}(x)
\end{aligned}
$$

is a morphism of complexes $(\widetilde{\Omega}, \Gamma(\mathcal{E})) \rightarrow(\Omega, \Gamma(\mathcal{H}))$. We note that $\mathfrak{f}$ has no image in $\Gamma(\mathcal{G})$, while $\left(\Omega^{\mathcal{G}}\right)^{-1} \mathcal{G \mathcal { E }} \phi^{\mathcal{E}}(x) \in \Gamma(\mathcal{F})$. That $\mathfrak{f} \widetilde{\Omega}=\Omega \mathfrak{f}$ follows from the identity

$$
\stackrel{\mathcal{F} \mathcal{E}}{\Omega}-\mathcal{F F}(\mathcal{G F})^{-1} \stackrel{\mathcal{G E}}{\Omega}=-(\mathcal{G F})^{-1} \Omega \mathcal{G E} \widetilde{\Omega}
$$

which in turn can be established by straightforward algebra using the nilpotency of $\Omega$ in the different subspaces. Furthermore, the map $\tilde{\mathfrak{f}}$ induced in cohomology can easily be shown to be an isomorphism.

One possibility to find a decomposition $\mathcal{H}=\mathcal{E} \oplus \mathcal{F} \oplus \mathcal{G}$ with $\stackrel{\mathcal{G F}}{\Omega}$ invertible is as follows. We suppose $\mathcal{H}$ to be equipped with an additional grading besides the ghost number,

$$
\mathcal{H}=\bigoplus_{i \geqslant 0} \mathcal{H}_{i}, \quad \operatorname{deg}\left(\mathcal{H}_{i}\right)=i
$$

extend the degree from $\mathcal{H}$ to $\mathcal{H}$-valued sections, and let the BRST operator $\Omega$ have the form

$$
\Omega=\Omega_{-1}+\Omega_{0}+\sum_{i \geqslant 1} \Omega_{i}, \quad \operatorname{deg}\left(\Omega_{i}\right)=i,
$$

with $\Omega_{i}: \Gamma(\mathcal{H})_{j} \rightarrow \Gamma(\mathcal{H})_{i+j} .{ }^{4}$

Proposition 3.6. Let $\Omega_{-1}$ be independent of $x$ and $x$-derivatives and let $\mathcal{H}=\mathcal{E} \oplus \mathcal{F} \oplus \mathcal{G}$ with $\operatorname{Ker} \Omega_{-1} \supset \mathcal{E} \cong H\left(\Omega_{-1}, \mathcal{H}\right)$ and $\mathcal{G}=\operatorname{Im} \Omega_{-1}$. Then $\stackrel{\mathcal{G F}}{\Omega}$ is algebraically invertible, and hence the system $(\Omega, \Gamma(\mathcal{H}))$ can be algebraically reduced to $(\widetilde{\Omega}, \Gamma(\mathcal{E}))$.

Proof. Because $\Omega_{-1}$ contains no $x$ dependence and no $x$-derivatives and because the space $\mathcal{H}$ is (graded) finite-dimensional, we have decomposition (3.9), where $\mathcal{E} \oplus \mathcal{G}=\operatorname{Ker} \Omega_{-1}$, $\mathcal{G}=\operatorname{Im} \Omega_{-1}$, and $\mathcal{F}$ is a complementary subspace, which is isomorphic to $\mathcal{G}$. We can

\footnotetext{
${ }^{4}$ In the case where equations of motion have the form $\Omega \Phi=0$ with $\Phi$ a collection of fields which are differential $p$-forms and $\Omega$ a flat covariant differential, the dynamical fields were identified with the cohomology of $\Omega_{-1}$ at form degree $p$ already in [24, 22].
} 
then construct the operator $\rho=\left(\stackrel{\mathcal{G F}}{\Omega}_{-1}\right)^{-1}$. Because $\stackrel{\mathcal{G F}}{\Omega}^{\mathcal{G} \mathcal{G}} \Omega_{-1}+\ldots$, it can be formally inverted order by order in the degree, with the inverse given by

$$
\left(\Omega^{\mathcal{G F}}\right)^{-1}=\sum_{n \geqslant 0}(-1)^{n} \rho\left[\left(\sum_{i \geqslant 0}^{\mathcal{G F}} \Omega_{i}\right) \rho\right]^{n} .
$$

If in addition the cohomology of $\Omega_{-1}$ is concentrated in one degree, then $\widetilde{\Omega}$ is the BRST operator induced by $\Omega_{0}$ in $\mathcal{E}, \widetilde{\Omega}=\Omega_{0}$. This follows because under the assumptions of Proposition 3.6 the degree of $(\Omega \mathcal{G})^{-1}$ is strictly positive, and hence so is the degree of the second term in the definition of $\widetilde{\Omega}$ in 3.12 , which therefore cannot contribute.

In fact, the invertibility of $\stackrel{\mathcal{G F}}{\Omega}$ implies the existence of contractible pairs of the form

$$
\Omega \widetilde{e}_{\alpha}=\widetilde{e}_{\beta} \widetilde{\Omega}_{\alpha}^{\beta}, \quad \Omega \widetilde{f}_{a}=\widetilde{g}_{a},
$$

but in a bigger space, namely the free $\mathcal{D}$-module generated by $\mathcal{H}$.

Indeed, we can consider the complex $(\Omega, \mathcal{D}(\mathcal{H}))$ with $\mathcal{D}(\mathcal{H})=\mathcal{H} \otimes \operatorname{Diff}(\mathcal{X})$ where $\operatorname{Diff}(\mathcal{X})$ is the algebra of differential operators on $\mathcal{X} \cdot \mathcal{D}(\mathcal{H})$ is a free $\operatorname{Diff}(\mathcal{X})$-module. The space $\Gamma(\mathcal{H})$ can be naturally identified with a subspace in $\mathcal{D}(\mathcal{H})$. In terms of components, if $\left(e_{A}\right)$ is a basis of $\mathcal{H}$, then elements of $\Gamma(\mathcal{H})$ are of the form $\phi(x)=e_{A} \phi^{A}(x)$ for some functions $\phi^{A}(x)$, while elements of $\mathcal{D}(\mathcal{H})$ are of the form $O\left(x, \frac{\partial}{\partial x}\right)=e_{A} O^{A}\left(x, \frac{\partial}{\partial x}\right)$, where $O^{A}\left(x, \frac{\partial}{\partial x}\right)$ are differential operators. With respect to $\left(e_{A}\right)$, the action of $\Omega$ in $\mathcal{D}(\mathcal{H})$ is defined as $\Omega O=e_{B} \Omega_{A}^{B} O^{A}$. A local frame $\left(\widehat{e}_{A}\right)$ for $\mathcal{D}(\mathcal{H})$ is a collection of elements of $\mathcal{D}(\mathcal{H})$ such that every element $O$ can be uniquely decomposed as $O=\widehat{e}_{A} \widehat{O}^{A}$ with $\widehat{O}^{A}$ differential operators. In particular, the basis $\left(e_{A}\right)$ of $\mathcal{H}$ induces a local frame of $\mathcal{D}(\mathcal{H})$. Given a frame $\left(e_{A}\right) \equiv\left(e_{\alpha}, f_{a}, g_{a}\right)$, we define a new frame $\left(\widetilde{e}_{A}\right)=\left(e_{B} A_{A}^{B}\right) \equiv\left(\widetilde{e}_{\alpha}, \widetilde{f}_{a}, \widetilde{g}_{a}\right)$, with $A_{A}^{B}$ invertible differential operators, through the invertible relations

$$
\begin{gathered}
\widetilde{e}_{\alpha}=e_{\alpha}-f_{b}\left((\stackrel{\mathcal{G F}}{\Omega})^{-1} \stackrel{\mathcal{G E}}{\Omega}\right)_{\alpha}^{b}, \\
\tilde{f}_{a}=f_{b}\left((\mathcal{G \mathcal { F }})^{-1}\right)_{a}^{b}, \\
\widetilde{g}_{a}=e_{\beta}\left(\mathcal{\mathcal { F }}(\stackrel{\mathcal{G F}}{\Omega})^{-1}\right)_{a}^{\beta}+f_{b}\left(\mathcal{\mathcal { F }}(\stackrel{\mathcal{G F}}{\Omega})^{-1}\right)_{a}^{b}+g_{a} .
\end{gathered}
$$

The expression for $\Omega$ in the new frame reduces to the Jordan form (3.19), as can be checked by using identity (3.15). We call the frame elements $\widetilde{f}_{a}, \widetilde{g}_{a}$ algebraically contractible pairs. The frame $\left(\widetilde{e}_{A}\right)$ can also be used to expand elements of $\Gamma(\mathcal{H}), \phi(x)=$ $e_{A} \phi^{A}(x)=\widetilde{e}_{A} \widetilde{\phi}^{A}(x)$, where $\widetilde{e}_{A}$ acts on $\widetilde{\phi}^{A}(x)$. The components $\widetilde{\phi}^{A}(x)$ are thus related to the components $\phi^{A}(x)$ and their derivatives according to $\widetilde{\phi}^{A}(x)=\left(A^{-1}\right)_{B}^{A} \phi^{B}(x)$. In Sec. 2.2 we have associated fields with (a local frame induced by) a basis $\left(e_{A}\right)$ of $\mathcal{H}$. Here, we are led to take a more general point of view and associate fields with elements 
of a local frame $\left(\widehat{e}_{A}\right)$ in $\mathcal{D}(\mathcal{H})$. At the field theory level, the fields associated with $\widehat{e}_{A}$ are related to those associated with $e_{A}$ through a linear invertible redefinition involving derivatives.

We have thus shown, by combining with Lemma $\mathbf{3 . 3}$

Proposition 3.7. After an invertible redefinition of the fields and their derivatives, generalized auxiliary fields are the fields associated with algebraically contractible pairs for $\Omega$ in $\mathcal{D}(\mathcal{H})$.

3.4. The action for an equivalence class of equations of motion. Starting from linear equations of motion (2.10), which can be written as

$$
\Omega_{B_{0}}^{A_{-1}} \psi^{(0) B_{0}}=0
$$

one can ask the question when are these equations equivalent to variational ones.

Usually, Eqs. (3.23) are defined to be equivalent to variational ones if there exist fieldindependent differential operators

$$
\omega_{A_{0} B_{-1}}=\omega_{A_{0} B_{-1}}^{0}+\omega_{A_{0} B_{-1}}^{\mu} \partial_{\mu}+\ldots
$$

with $\omega_{A_{0} B_{-1}}^{0}$ invertible, such that $\omega_{A_{0} B_{-1}}\left(\Omega_{B_{0}}^{A_{-1}} \psi^{(0) B_{0}}\right)$ is given by Euler-Lagrange derivatives of some quadratic Lagrangian. Standard results on the inverse problem of the calculus of variations (see, e.g., [36, 47, 37]) show that this is the case if and only if the operator $\Omega_{A_{0} B_{0}} \equiv \omega_{A_{0} C_{-1}} \circ \Omega_{B_{0}}^{C_{-1}}$ is formally self-adjoint. Here, formal self-adjointness means that

$$
\Omega_{A_{0} B_{0}}=(-1)^{\left|A_{0}\right|\left|B_{0}\right|} \Omega_{A_{0}}^{+C_{-1}} \circ \omega_{B_{0} C_{-1}}^{+},
$$

where the adjoint + of an operator $O=\sum_{k=0} O^{\mu_{1} \ldots \mu_{k}} \partial_{\mu_{1}} \ldots \partial_{\mu_{k}}$ is defined as $O^{+} f \equiv$ $\sum_{k=0}(-1)^{k} \partial_{\mu_{1}} \ldots \partial_{\mu_{k}}\left[O^{\mu_{1} \ldots \mu_{k}} f\right]$ for any local function $f$. (We note that the adjoint here is a priori not related to the conjugation defined on $\mathcal{H}$.) Whenever such an $\Omega_{A_{0} B_{0}}$ exists, the action for physical fields can be written as

$$
\boldsymbol{S}^{\mathrm{ph}}\left[\psi^{(0)}\right]=\frac{1}{2} \int d^{d} x \psi^{(0) A_{0}} \Omega_{A_{0} B_{0}} \psi^{(0) B_{0}} .
$$

Similarly, one can ask when the BRST differential $s$ in (3.1) is canonically generated by some quadratic master action $S$ with respect to some invertible field-independent antibracket. This is the case if and only if there exist field-independent differential operators

$$
\omega_{A_{k} B_{-k-1}}=\omega_{A_{k} B_{-k-1}}^{0}+\omega_{A_{k} B_{-k-1}}^{\mu} \partial_{\mu}+\ldots,
$$

with $\omega_{A_{k} B_{-k-1}}^{0}$ invertible and $\omega_{A_{k} B_{-k-1}}=(-1)^{1+\left|A_{k}\right|\left|B_{-k-1}\right|} \omega_{B_{-k-1} A_{k}}^{+}$, such that the differential operator $\Omega_{A_{k} B_{-k}} \equiv \omega_{A_{k} C_{-k-1}} \circ \Omega_{B_{-k}}^{C_{-k-1}}$ is formally self-adjoint. In this case, the 
master action is given by

$$
\boldsymbol{S}[\psi]=\frac{1}{2} \int d^{d} x \sum_{k} \psi_{k}^{A_{k}} \Omega_{A_{k} B_{-k}} \psi_{-k}^{B_{-k}},
$$

while the antibracket is determined through $\left(\psi^{A_{k}}(x), \psi^{B_{-k-1}}(y)\right)=\omega^{A_{k} B_{-k-1}} \delta(x, y)$. Furthermore, if a compatible complex structure exists on $\mathcal{H}$, the symplectic structure $\omega_{A_{k} B_{-k-1}}$ determines a Hermitian inner product on $\Gamma(\mathcal{H})$.

We see at this stage that the setting in Sec. 2 can actually be generalized in the Hermitian inner product should be allowed to contain space-time derivatives.

In Secs. 3.2 and 3.3 we have discussed when two linear non-Lagrangian theories determined by the corresponding BRST operators are related via elimination of generalized auxiliary fields. Of course, such theories must be considered equivalent. It is therefore natural to address the problem of the existence of a variational principle with this more general notion of equivalence taken into account, i.e., to consider Eqs. (3.23) equivalent to variational ones if, after elimination or introduction of generalized auxiliary fields, they are equivalent to variational ones in the sense defined above. Similarly, a theory defined by a BRST differential $s$ should be considered as equivalent to one with a canonically generated $s$ if, after elimination or introduction of generalized auxiliary fields, there exists a symplectic form making the BRST operator formally self-adjoint.

At the first-quantized level, the situation where only one of two equivalent theories admits a Lagrangian manifests itself in the existence of two equivalent BRST first-quantized systems $(\Omega, \Gamma(\mathcal{H}))$ and $(\widetilde{\Omega}, \Gamma(\widetilde{\mathcal{H}}))$ such that a Hermitian inner product on the entire space making the BRST operator Hermitian is known only for one of them. For instance, suppose that we are in the setting of Proposition 3.6. where $\widetilde{\mathcal{H}}=\mathcal{E}=H\left(\Omega_{-1}, \mathcal{H}\right)$, and assume $\widetilde{\mathcal{H}}$ to be equipped with an inner product that makes $\widetilde{\Omega}$ Hermitian. In this case, we can conclude directly at the first-quantized level that the equations $\Omega \Psi^{(0)}=0$ are equivalent to Lagrangian equations $\widetilde{\Omega} \widetilde{\Psi}^{(0)}=0$ via elimination of generalized auxiliary fields. Examples of this type occur in the following sections.

3.5. First-quantized description of the Fronsdal Lagrangian. As an application of our approach, we show how a simple first-quantized BRST system gives rise to the sum of the Fronsdal Lagrangians for free massless higher-spin fields [4]. The system with which we start was proposed in [6, 7] (see also [8]).

The variables are $x^{\mu}, p_{\mu}, a^{\mu}, a^{\dagger \mu}$, where $\mu=1, \ldots, d$. Classically, $x^{\mu}$ and $p_{\mu}$ correspond to coordinates on $T^{*} X$ and $a^{\mu}, a^{\dagger \mu}$ correspond to internal degrees of freedom. After quantization, they satisfy the canonical commutation relations

$$
\left[p_{\nu}, x^{\mu}\right]=-\imath \delta_{\nu}^{\mu}, \quad\left[a^{\mu}, a^{\dagger \nu}\right]=\eta^{\mu \nu}
$$

We assume that $x^{\mu}, p_{\mu}$ are Hermitian, $\left(x^{\mu}\right)^{\dagger}=x^{\mu}, p_{\mu}^{\dagger}=p_{\mu}$, while $a^{\mu}$ and $a^{\dagger \mu}$ are interchanged by Hermitian conjugation. 
The constraints of the system are

$$
\mathcal{L} \equiv \eta^{\mu \nu} p_{\mu} p_{\nu}=0, \quad \mathcal{S} \equiv p_{\mu} a^{\mu}=0, \quad \mathcal{S}^{\dagger} \equiv p_{\mu} a^{\dagger \mu}=0 .
$$

For the ghost pairs $(\theta, \mathcal{P}),\left(c^{\dagger}, b\right)$, and $\left(c, b^{\dagger}\right)$ corresponding to each of these constraints, we take the canonical commutation relations in the form ${ }^{5}$

$$
[\mathcal{P}, \theta]=-\imath, \quad\left[c, b^{\dagger}\right]=1, \quad\left[b, c^{\dagger}\right]=-1 .
$$

The ghost-number assignments are

$$
\operatorname{gh}(\theta)=\operatorname{gh}(c)=\operatorname{gh}\left(c^{\dagger}\right)=1, \quad \operatorname{gh}(\mathcal{P})=\operatorname{gh}(b)=\operatorname{gh}\left(b^{\dagger}\right)=-1 .
$$

The BRST operator is then given by

$$
\Omega_{0}=\theta \mathcal{L}+c^{\dagger} \mathcal{S}+\mathcal{S}^{\dagger} c-\imath \mathcal{P} c^{\dagger} c .
$$

The representation space is given by functions of $x^{\mu}$ (on which $p_{\mu}$ acts as $-i \frac{\partial}{\partial x^{\mu}}$ ) with values in the "internal space" $\mathcal{H}_{0}$. The latter is the tensor product of the space $\mathcal{H}_{\theta, \mathcal{P}}$ of functions in $\theta$ (coordinate representation for $(\theta, \mathcal{P})$ ) and the Fock spaces for $\left(a_{\mu}^{\dagger}, a^{\mu}\right)$, $\left(c^{\dagger}, b\right)$, and $\left(c, b^{\dagger}\right)$ with the vacuum conditions

$$
a^{\mu}|0\rangle=b|0\rangle=c|0\rangle=0 .
$$

The inner product in the space $\mathcal{H}_{0}$ is denoted by $\langle\cdot, \cdot\rangle$. It is given by the tensor product of the standard Fock space inner product and the inner product $\langle,\rangle_{\theta, \mathcal{P}}$ in $\mathcal{H}_{\theta, \mathcal{P}}$ determined by $\langle\theta, 1\rangle_{\theta, \mathcal{P}}=1$, where $\theta$ and 1 are basis elements.

In accordance with the homogeneity degree in $\theta$, the BRST operator decomposes as

$$
\Omega_{0,-1}=-\imath \mathcal{P} c^{\dagger} c, \quad \Omega_{0,0}=c^{\dagger} \mathcal{S}+\mathcal{S}^{\dagger} c, \quad \Omega_{0,1}=\theta \mathcal{L} .
$$

The assumptions of Proposition 3.6 are then satisfied. In the present case, we have that the states with the ghost dependence $\theta b^{\dagger}$ and $c^{\dagger}$ form contractible pairs for $\Omega_{0,-1}$, while all other states are representatives of cohomology classes. Furthermore, because $\theta b^{\dagger}$ carries ghost number 0 , it follows from Proposition 3.1 that the associated fields are ordinary auxiliary fields.

The ghost-number-zero component of the string field is

$$
\Psi^{(0)}=\Phi-\imath \theta b^{\dagger} C+c^{\dagger} b^{\dagger} D
$$

where

$$
\begin{aligned}
& \Phi=\sum_{s=0}^{\infty} \frac{1}{s !} a^{\dagger \mu_{1}} \ldots a^{\dagger \mu_{s}}|0\rangle \stackrel{s}{\varphi}_{\mu_{1} \ldots \mu_{s}}, \\
& C=\sum_{s=1}^{\infty} \frac{1}{(s-1) !} a^{\dagger \mu_{1}} \ldots a^{\dagger \mu_{s-1}}|0\rangle{\stackrel{s}{c_{\mu_{1} \ldots \mu_{s-1}}},}^{\infty},
\end{aligned}
$$

\footnotetext{
${ }^{5}$ We use the "super" convention that $(a b)^{\dagger}=(-1)^{|a||b|} b^{\dagger} a^{\dagger}$.
} 


$$
D=\sum_{s=2}^{\infty} \frac{1}{(s-2) !} a^{\dagger \mu_{1}} \ldots a^{\dagger \mu_{s-2}}|0\rangle \stackrel{s}{d}_{\mu_{1} \ldots \mu_{s-2}},
$$

with totally symmetric tensor fields $\stackrel{s}{\varphi}, \stackrel{s}{c}$, and $\stackrel{s}{d}$. The expression for physical action (2.16), with $\Omega$ replaced by $\Omega_{0}$, then becomes

$$
\begin{aligned}
\boldsymbol{S}^{\mathrm{ph}}[\varphi, c, d]=-\frac{1}{2} \int d^{d} x[ & \langle\Phi, \mathcal{L} \Phi\rangle+\imath\left\langle\Phi, \mathcal{S}^{\dagger} C\right\rangle-\imath\langle C, \mathcal{S} \Phi\rangle \\
& \left.+\imath\left\langle C, \mathcal{S}^{\dagger} D\right\rangle-\imath\langle D, \mathcal{S} C\rangle+\langle C, C\rangle-\langle D, \mathcal{L} D\rangle\right]
\end{aligned}
$$

where $\langle\cdot, \cdot\rangle$ is the inner product on the Fock space of $a^{\mu}, a^{\dagger \mu}$ alone. Eliminating the auxiliary fields contained in $C$ and assuming reality conditions in accordance with which the fields $\varphi_{\mu_{1}, \ldots, \mu_{s}}$ and $d_{\mu_{1}, \ldots, \mu_{s-2}}$ are real, we arrive at

$$
\begin{aligned}
\boldsymbol{S}^{\mathrm{ph}}[\varphi, d]=-\int d^{d} x\left[\frac{1}{2}\langle\Phi, \mathcal{L} \Phi\rangle-\right. & \frac{1}{2}\langle\mathcal{S} \Phi, \mathcal{S} \Phi\rangle+ \\
& \left.+\left\langle D, \mathcal{S}^{2} \Phi\right\rangle-\langle D, \mathcal{L} D\rangle-\frac{1}{2}\langle\mathcal{S} D, \mathcal{S} D\rangle\right]
\end{aligned}
$$

In component form, we rewrite this as

$$
\begin{aligned}
& \boldsymbol{S}^{\mathrm{ph}}[\varphi, d]=-\sum_{s=0}^{\infty} \frac{1}{s !} \int d^{d} x\left[\frac{1}{2}\left(\partial_{\mu} \stackrel{s}{\varphi}, \partial^{\mu} \stackrel{s}{\varphi}\right)-\frac{s}{2}(\partial \cdot \stackrel{s}{\varphi}, \partial \cdot \stackrel{s}{\varphi})-\right. \\
& \left.-s(s-1)(\stackrel{s}{d}, \partial \cdot \partial \cdot \stackrel{s}{\phi})-s(s-1)\left(\partial_{\mu} \stackrel{s}{d}, \partial^{\mu} \stackrel{s}{d}\right)-\frac{s(s-1)(s-2)}{2}(\partial \cdot \stackrel{s}{d}, \partial \cdot \stackrel{s}{d})\right]
\end{aligned}
$$

where $(\partial \cdot \stackrel{s}{\varphi})_{\mu_{1}, \ldots, \mu_{s-1}}=s \partial^{\nu} \stackrel{s}{\varphi}_{\nu \mu_{1}, \ldots, \mu_{s-1}}$ and the standard inner product $(\cdot, \cdot)$ for symmetric tensors is introduced, e.g., for 2-tensors $a, b$ we have $(a, b)=\eta^{\mu \rho} \eta^{\nu \sigma} a_{\mu \nu} b_{\rho \sigma}$.

The last expression is almost the sum of the Fronsdal Lagrangians (which can of course be recognized directly in (3.39). To obtain the Fronsdal action from (3.39), it remains to impose the conditions that the $\stackrel{s}{d}_{\mu_{1}, \ldots, \mu_{s-2}}$ fields are half the traces of $\stackrel{s}{\varphi}_{\mu_{1}, \ldots, \mu_{s}}$ and that the $\stackrel{s}{\varphi}_{\mu_{1}, \ldots, \mu_{s}}$ fields are double-traceless. Remarkably, this can be done at the BRST firstquantized level. For this, we impose the additional constraint

$$
T \equiv \eta_{\mu \nu} a^{\mu} a^{\nu}=0
$$

and let $\xi, \pi$, with

$$
\operatorname{gh}(\xi)=1, \quad \operatorname{gh}(\pi)=-1,
$$

denote the corresponding ghost pair, represented in the space $\mathcal{H}_{\xi, \pi}$ of functions of $\xi$. The extended BRST operator is then given by

$$
\Omega=\Omega_{-1}+\Omega_{0}, \quad \Omega_{-1}=\xi \mathcal{T}, \quad \mathcal{T}=\eta_{\mu \nu} a^{\mu} a^{\nu}+2 c b,
$$

with $\Omega_{0}$ defined in (3.33). With the degree taken to be minus the homogeneity in $\xi$, the conditions of Proposition 3.6 are satisfied. In fact, the cohomology $H\left(\Omega_{-1}, \mathcal{H}_{0} \otimes \mathcal{H}_{\xi, \pi}\right)$ 
can be identified with the subspace $\mathcal{E}$ of $\xi$-independent vectors $\psi$ satisfying $\mathcal{T} \psi=0$, because any symmetric tensor can be written as the trace of a symmetric tensor.

It now follows from Proposition 3.6 that after elimination of the generalized auxiliary fields, the physical action for the BRST system described by $\Omega$ becomes

$$
\boldsymbol{S}_{F}^{\mathrm{ph}}=-\frac{1}{2} \int d^{d} x\left\langle\widetilde{\Psi}^{(0)}, \widetilde{\Omega} \widetilde{\Psi}^{(0)}\right\rangle
$$

where $\widetilde{\Psi}$ is the string field associated with $\mathcal{E}$ and $\widetilde{\Omega}$ is the restriction of $\Omega_{0}$ to $\mathcal{E}$-valued sections. Again, the fields associated with states with the ghost dependence $\theta b^{\dagger}$ are ordinary auxiliary fields. The string field $\widetilde{\Psi}^{(0)}(x)$ can be identified with the solution of the constraint

$$
\mathcal{T} \Psi^{(0)}=0
$$

for the string field of form (3.36). The solution is easy to find in terms of the fields $\Phi, C$, and $D$,

$$
T \Phi=2 D, \quad T C=0, \quad T D=0 .
$$

Clearly, in terms of the tensor fields introduced in (3.37), $T$ is the operation of taking

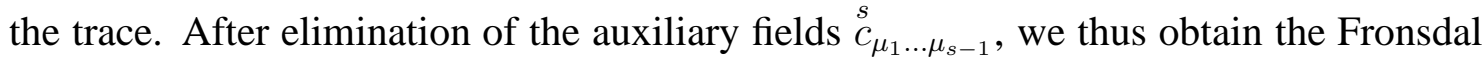
Lagrangians.

\section{Remarks.}

(i) We note that $\Omega$ is no longer Hermitian in the standard inner product on $\mathcal{H}_{0} \otimes \mathcal{H}_{\xi, \pi}$. This is the reason why the system described by $\Omega$ is non-Lagrangian. Nevertheless, as we have just shown, it is equivalent to a Lagrangian system in the sense of the previous subsection.

(ii) In [9] (see also [48] for a recent discussion), the Fronsdal Lagrangians were obtained by imposing the constraint $\mathcal{T}$ directly on the string field without introducing a corresponding ghost pair and including it in a BRST operator. Other approaches to obtain the Fronsdal Lagrangians can be found in [13, 16].

(iii) To describe the Fronsdal Lagrangian for a particular spin $s$, the occupation-number constraint

$$
N_{s} \equiv a_{\mu}^{\dagger} a^{\mu}-c^{\dagger} b+b^{\dagger} c-s=0
$$

must be imposed in addition to $\mathcal{T}=0$. Trying to incorporate $N_{s}$ with some ghosts $\xi, \pi$ into the BRST operator $\Omega_{0}$ in the same way as we did for $\mathcal{T}$, we see that each state annihilated by $N_{s}$ appears twice in the cohomology, once multiplied by the ghost $\xi$ and once without it. This is why the individual Fronsdal Lagrangians cannot be directly obtained by eliminating generalized auxiliary fields, not even on the level of the equations of motion. 
A way out is to treat the constraint $N_{s}=0$ in the same way as the level-matching condition in closed string field theory [49]: the doubling is eliminated by requiring the string field to be annihilated by $\pi$, and the action is obtained by regularizing the inner product with an insertion of $\xi$. An alternative is not to introduce ghost pairs for $\mathcal{T}$ and $N_{s}$ in the first place and impose both $\mathcal{T}=0$ and $N_{s}=0$ as constraints on the string field. This can be done consistently because $\left[N_{s}, \mathcal{T}\right]=-2 \mathcal{T}$ and both constraints commute with $\Omega_{0}$.

\section{THE FIRST-ORDER PARENT SYSTEM}

We now show how to replace a BRST first-quantized system by an equivalent extended system that is first-order in space-time derivatives. Such a construction is related to the conversion of second-class constraints [50, 51] or a version of Fedosov quantization [52] (see [53, 54] for a unified description of both). The field theory associated with the extended system is then also first-order and is reduced to the original one by elimination of generalized auxiliary fields. A different reduction of the extended theory by the elimination of another collection of generalized auxiliary fields gives rise to a first-order system representing the unfolded form [18, 20] of the original field theory. This last reduction is explicitly illustrated in the case of the Klein-Gordon and Fronsdal equations.

4.1. Extended first-quantized system and parent field theory. We recall the classical constrained system on $T^{*} X \times B$ described at the beginning of Sec. 2.1 where $x^{\mu}, p_{\mu}, \mu=$ $1, \ldots, d$, are coordinates on $T^{*} \mathcal{X}=T^{*} \mathbb{R}^{d}$. We embed the phase space $T^{*} X \times B$ into the extended phase space $T^{*}(T X) \times B$ as the second-class constraint surface determined by

$$
p_{\mu}-p_{\mu}^{y}=0, \quad y^{\mu}=0 .
$$

In terms of coordinates $x^{\mu}, y^{\nu}, p_{\mu}, p_{\nu}^{y}$ on $T^{*}(T X)$, the corresponding canonical Poisson brackets are

$$
\left\{p_{\mu}, x^{\nu}\right\}=-\delta_{\mu}^{\nu}, \quad\left\{p_{\mu}^{y}, y^{\nu}\right\}=-\delta_{\mu}^{\nu} .
$$

Constraints (4.1) are then indeed second-class. The Poisson bracket on $T^{*}(T X) \times B$ is given by the product of the Poisson brackets of the factors. In the BRST formalism, these constraints can be taken into account by treating only the first set as first-class constraints. This requires extending the phase space further by ghosts $\mathcal{C}^{\mu}$ and ghost momenta $\mathcal{P}_{\mu}$ with the Poisson bracket $\left\{\mathcal{P}_{\mu}, \mathcal{C}^{\nu}\right\}=-\delta_{\mu}^{\nu}$.

At the quantum level, the phase-space coordinates become operators with the nonzero commutation relations

$$
\left[p_{\nu}, x^{\mu}\right]=-\imath \delta_{\nu}^{\mu}, \quad\left[p_{\nu}^{y}, y^{\mu}\right]=-\imath \delta_{\nu}^{\mu}, \quad\left[\mathcal{P}_{\nu}, \mathcal{C}^{\mu}\right]=-\imath \delta_{\nu}^{\mu} .
$$

As in Sec. 2.1, we here assume that the internal space $B$ is quantized, with the representation space denoted by $\mathcal{H}$. The BRST operator corresponding to constraints (4.1) is 


$$
\Omega^{X}=\mathcal{C}^{\mu}\left(p_{\mu}-p_{\mu}^{y}\right)
$$

All the constraints, including the original ones on $T^{*} X \times B$ (Eqs. (2.11), are taken into account by the "total" BRST operator

$$
\Omega^{\mathrm{T}}=\Omega^{x}+\Omega^{y}
$$

where $\Omega^{y}$ is constructed as a formal power series in $y^{\mu}$ and $p_{\mu}^{y}$ such that

$$
\left[\Omega^{x}+\Omega^{y}, \Omega^{x}+\Omega^{y}\right]=0,\left.\quad \Omega^{y}\right|_{y=0, p_{\mu}-p_{\mu}^{y}=0}=\Omega .
$$

The construction of $\Omega^{\mathrm{T}}$ is an adapted version of the general method of including original first-class constraints in an extended system, see [51].

In what follows, we assume BRST operator $\Omega$ to be x-independent, and hence $\Omega^{y}$ can be taken to be just $\Omega$ with $p_{\mu}$ replaced by $p_{\mu}^{y}$.

The space of quantum states of the extended system is chosen to be the space $\Gamma\left(\mathcal{H}^{\mathrm{T}}\right)$ of $\mathcal{H}^{\mathrm{T}}$-valued sections, with $\mathcal{H}^{\mathrm{T}}=\mathcal{H} \otimes \mathrm{S}\left(T^{*}\right) \otimes \wedge\left(T^{*}\right)$, where $T^{*}$ is the cotangent space to $X$ and where $S$ and $\wedge$ denote the symmetric and skew-symmetric tensor algebras. In other words, states can be identified with "functions" of the form $\phi=\phi(x, y, \mathcal{C})=$ $e_{A} \phi^{A}(x, y, \mathcal{C})$, where $\left(e_{A}\right)$ is a basis in $\mathcal{H}$ and each $\phi^{A}$ is a function in $x$, a formal power series in the commuting variables $y^{\mu}$, and a polynomial in the anticommuting variables $\mathcal{C}^{\mu}, \mu=1, \ldots, d$. The momenta $p_{\mu}, p_{\mu}^{y}$, and the ghost momenta $\mathcal{P}_{\mu}$ act as $-\imath \frac{\partial}{\partial x^{\mu}}$, $-\imath \frac{\partial}{\partial y^{\mu}}$, and $-\imath \frac{\partial}{\partial \mathcal{C}^{\mu}}$ respectively; as noted above, the operators corresponding to the internal degrees of freedom are represented on $\mathcal{H}$. The basis elements in $\mathcal{H}^{\mathrm{T}}$ are given by

$$
e_{A}^{(\mu)[\nu]}=e_{A} \otimes y^{\mu_{1}} \ldots y^{\mu_{n}} \mathcal{C}^{\nu_{1}} \ldots \mathcal{C}^{\nu_{l}},
$$

where $(\mu)$ and $[\nu]$ denote a collection of respectively symmetrized and antisymmetrized indices.

Following Sec. 2.1 we construct the field theory for the extended system $\left(\Omega^{\mathrm{T}}, \Gamma\left(\mathcal{H}^{\mathrm{T}}\right)\right)$ in terms of the string field

$$
\Psi^{\mathrm{T}}(x)=e_{A}^{(\mu)[\nu]} \psi_{(\mu)[\nu]}^{A}(x) .
$$

The total BRST operator $\Omega^{\mathrm{T}}$ acts on $\Psi^{\mathrm{T}}$ as

$$
\Omega^{\mathrm{T}} \Psi^{\mathrm{T}}(x)=-\imath\left(\boldsymbol{d}+\sigma+\imath \Omega^{y}\right) \Psi^{\mathrm{T}}(x),
$$

where

$$
\boldsymbol{d}=\mathcal{C}^{\mu} \frac{\partial}{\partial x^{\mu}}, \quad \sigma=-\mathcal{C}^{\mu} \frac{\partial}{\partial y^{\mu}}
$$

(and we recall that $\Omega^{y}$ is given by $\Omega$ with the momenta $p_{\mu}$ replaced by $p_{\mu}^{y}$, which act as $\left.-\imath \frac{\partial}{\partial y^{\mu}}\right)$. The BRST differential $s^{\mathrm{T}}$ is then determined by $s^{\mathrm{T}} \Psi^{\mathrm{T}}=\Omega^{\mathrm{T}} \Psi^{\mathrm{T}}$. 
In what follows, the field theory determined by $s^{\mathrm{T}}$ and $\Psi^{\mathrm{T}}$ is called the parent theory. Its role is to produce apparently different theories via elimination of different collections of generalized auxiliary fields. By the above analysis, all these theories are guaranteed to be just equivalent representations of the same dynamics. Two of its reductions are studied in Secs. 4.2 and 4.3 .

4.2. Reduction to the original system. We now show that via the elimination of generalized auxiliary fields, the parent theory determined by the BRST differential $s^{T}$ is equivalent to the original theory determined by the BRST differential $s \Psi^{A}=(\Omega \Psi)^{A}$.

Proposition 4.1. The parent system $\left(\Omega^{\mathrm{T}}, \Gamma\left(\mathcal{H}^{\mathrm{T}}\right)\right)$ can be algebraically reduced to the original, $y^{\mu}$ - and $\mathcal{C}^{\mu}$-independent system $(\Omega, \Gamma(\mathcal{H}))$.

Proof. The proof consists in (i) constructing a decomposition

$$
\mathcal{H}^{\mathrm{T}}=\mathcal{E} \oplus \mathcal{F} \oplus \mathcal{G}
$$

where $\mathcal{E}$ is isomorphic to $\mathcal{H}$ with $\stackrel{\mathcal{G} \mathcal{F}}{\Omega^{\mathrm{T}}}$ invertible, and (ii) showing that $\widetilde{\Omega^{\mathrm{T}}}$ is mapped to $\Omega$ under the isomorphism.

To construct the required decomposition of $\mathcal{H}^{\mathrm{T}}$, we use Proposition $\mathbf{3 . 6}$ with the underlying grading chosen as follows. Our assumptions imply that $\Omega^{y}$ is a polynomial in the $\frac{\partial}{\partial y^{\mu}}, \mu=1, \ldots, d$. Let $\ell$ be the maximum power of $\frac{\partial}{\partial y^{\mu}}$ involved in $\Omega^{y}$. The grading is given by (homogeneity degree in $y)+\ell$ (target-space ghost number), where the targetspace ghost number is the ghost number that does not count the number of $\mathcal{C}^{\mu}$ 's. This grading is assumed to be bounded from below and, without loss of generality, the bound can be taken to be zero.

With respect to this grading (indicated by a subscript), the BRST operator $\Omega^{\mathrm{T}}$ decomposes as in (3.17) with $\imath \Omega_{-1}^{\mathrm{T}}=\sigma$. It is then obvious that the cohomology of $\Omega_{-1}^{\mathrm{T}}$ in $\mathcal{H}^{\mathrm{T}}$ can be identified with $\mathcal{H} \subset \mathcal{H}^{\mathrm{T}}$.

To complete decomposition 4.11, we introduce the operators

$$
\rho=-y^{\mu} \frac{\partial}{\partial \mathcal{C}^{\mu}}, \quad \tau=y^{\mu} \frac{\partial}{\partial x^{\mu}}, \quad N=\mathcal{C}^{\mu} \frac{\partial}{\partial \mathcal{C}^{\mu}}+y^{\mu} \frac{\partial}{\partial y^{\mu}}
$$

acting in $\mathcal{H}^{\mathrm{T}}$ and set

$$
\mathcal{F}=\rho \mathcal{H}^{\mathrm{T}}, \quad \mathcal{G}=\sigma \mathcal{H}^{\mathrm{T}}
$$

By Proposition 3.6 the operator $\Omega^{\mathrm{G}}$ is algebraically invertible, and therefore the parent system $\left(\Omega^{\mathrm{T}}, \Gamma\left(\mathcal{H}^{\mathrm{T}}\right)\right)$ can be algebraically reduced to the system $\left(\widetilde{\Omega^{\mathrm{T}}}, \Gamma(\mathcal{H})\right)$.

It remains to calculate $\widetilde{\Omega^{\mathrm{T}}}$. For this, we introduce the grading with respect to the space-time ghost number, which is determined by the operator $\mathcal{C}^{\mu} \frac{\partial}{\partial \mathcal{C}^{\mu}}$. With this grading 
indicated by a superscript, we have the decomposition

where it is readily seen that

$$
\Omega^{\mathrm{T}}=\Omega_{-1}^{1}+\Omega_{0}^{1}+\sum_{i=0}^{\ell} \Omega_{i}^{0}
$$

$$
\imath \Omega_{-1}^{1}=\sigma, \quad \imath \Omega_{0}^{1}=\boldsymbol{d} .
$$

Moreover, $\Omega_{i}^{0}=\Omega_{[\ell-i]}^{y}$, where $\Omega_{[m]}^{y}$ is the term in $\Omega^{y}$ that is homogeneous of degree $m$ in $\frac{\partial}{\partial y^{\mu}}$ and we set $\Omega^{0}=\sum_{i=0}^{\ell} \Omega_{i}^{0}$.

The space $\mathcal{H}^{\mathrm{T}}$ decomposes accordingly,

$$
\mathcal{H}^{\mathrm{T}}=\bigoplus_{i \geqslant 0} \bigoplus_{j=0}^{d}\left(\mathcal{H}^{\mathrm{T}}\right)_{i}^{j}
$$

and it follows that

$$
\mathcal{E}=\bigoplus_{i \geqslant 0} \mathcal{E}_{i \ell}^{0}
$$

We refer to (elements of) $\left(\mathcal{H}^{\mathrm{T}}\right)_{i}^{j}$ as having the bidegree $\left(\begin{array}{l}j \\ i\end{array}\right)$.

To construct $\widetilde{\Omega^{\mathrm{T}}}$, we note that

$$
\stackrel{\mathcal{E} \mathcal{E}}{\Omega^{\mathrm{T}}}=\Omega_{\ell}^{0}=\Omega_{[0]}^{y}, \quad \stackrel{\mathcal{G} \mathcal{E}}{\Omega^{\mathrm{T}}}=\imath \Omega_{0}^{1}=\boldsymbol{d} .
$$

We also note that $N$ is invertible on the image of $\rho$ and on the image of $\sigma$. According to (3.18), the operator $\left(\Omega^{\mathcal{F}}\right)^{-1}: \mathcal{G} \rightarrow \mathcal{F}$ is given by

$$
\begin{aligned}
\left(\imath \Omega^{\mathcal{F}}\right. & -1 \\
\boldsymbol{\phi}^{\mathcal{G}} & =\left[N^{-1} \rho-N^{-1} \rho\left(\boldsymbol{d}+\imath \Omega^{0}\right) N^{-1} \rho\right. \\
& \left.+N^{-1} \rho\left(\boldsymbol{d}+\imath \Omega^{0}\right) N^{-1} \rho\left(\boldsymbol{d}+\imath \Omega^{0}\right) N^{-1} \rho-\ldots\right] \boldsymbol{\phi}^{\mathcal{G}}, \quad \forall \boldsymbol{\phi}^{\mathcal{G}} \in \Gamma(\mathcal{G})
\end{aligned}
$$

and is a two-sided inverse of $\imath \Omega^{\mathrm{G}}$. A simple argument based on grading shows that on any $\phi^{\mathcal{E}}$ in $\Gamma(\mathcal{E})$,

$$
\begin{array}{r}
\left(\imath \Omega^{\mathrm{G}}\right)^{-1} \imath \stackrel{\mathcal{G} \mathcal{E}}{\Omega^{\mathrm{T}}} \boldsymbol{\phi}^{\mathcal{E}}=\left(N^{-1} \rho-N^{-1} \rho \boldsymbol{d} N^{-1} \rho+N^{-1} \rho \boldsymbol{d} N^{-1} \rho \boldsymbol{d} N^{-1} \rho-\ldots\right) \boldsymbol{d} \boldsymbol{\phi}^{\mathcal{E}} \\
=\sum_{n \geqslant 1}(-1)^{n-1}\left(N^{-1} \rho \boldsymbol{d}\right)^{n} \boldsymbol{\phi}^{\mathcal{E}}
\end{array}
$$

The term $\left(N^{-1} \rho \boldsymbol{d}\right)^{n}$ increases the bidegree by $\left(\begin{array}{l}0 \\ n\end{array}\right)$. Because $\mathcal{E}$ has bidegree $\left(\begin{array}{l}0 \\ i \ell\end{array}\right)$ (see (4.17), it follows that

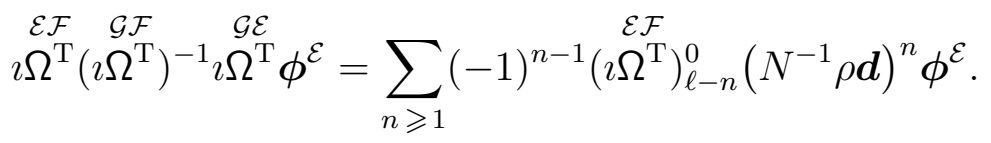


Noting that $\left(\Omega^{\mathcal{E}}\right)_{\ell-n}^{0}=\Omega_{\ell-n}^{0}=\Omega_{[n]}^{y}$, we finally evaluate (3.12) as

$$
\widetilde{\Omega^{\mathrm{T}}} \boldsymbol{\phi}^{\mathcal{E}}=\Omega_{[0]}^{y}+\sum_{n=1}^{\ell}(-1)^{n} \Omega_{[n]}^{y}\left(N^{-1} \rho \boldsymbol{d}\right)^{n} \boldsymbol{\phi}^{\mathcal{E}} .
$$

Rearranging the operators in this formula using that $\rho \mathcal{E}=0$ and $[\rho, \boldsymbol{d}]=-\tau$, we obtain

$$
\widetilde{\Omega}^{\mathrm{T}} \boldsymbol{\phi}^{\mathcal{E}}=\sum_{n=0}^{\ell} \frac{1}{n !} \Omega_{[n]}^{y} \tau^{n} \boldsymbol{\phi}^{\mathcal{E}} .
$$

For $n \geqslant 1$, moreover, $\Omega_{[n]}^{y} \phi^{\mathcal{E}}=0$ and $\Omega_{[n]}^{y} \tau^{n} \phi^{\mathcal{E}}=n ! \Omega_{[n]} \phi^{\mathcal{E}}$, where $\Omega_{[n]}$ is the term in $\Omega$ that is homogeneous of degree $n$ in $\frac{\partial}{\partial x^{\mu}}$. Therefore, $\widetilde{\Omega}^{\mathrm{T}} \phi^{\mathcal{E}}=\Omega \phi^{\mathcal{E}}$.

4.3. Reduction to the unfolded formalism. Taking another reduction of the parent system $\left(\Omega^{\mathrm{T}}, \Gamma\left(\mathcal{H}^{\mathrm{T}}\right)\right)$ to the subspace $\mathcal{E} \cong H\left(\Omega^{y}, \mathcal{H}^{\mathrm{T}}\right)$, we now construct the unfolded form of the original system.

Proposition 4.2. The parent system $\left(\Omega^{\mathrm{T}}, \Gamma\left(\mathcal{H}^{\mathrm{T}}\right)\right)$ can be algebraically reduced to $\left(\widetilde{\Omega}^{\mathrm{T}}\right.$, $\Gamma(\mathcal{E}))$ with $\mathcal{E} \cong H\left(\Omega^{y}, \mathcal{H}^{\mathrm{T}}\right)$.

Proof. To prove this, we take minus the target-space ghost number (defined in the paragraph after Eq. (4.11) as the degree. The BRST operator $\Omega^{\mathrm{T}}$ in (4.5) decomposes as $\Omega_{-1}^{\mathrm{T}}=\Omega^{y}, \Omega_{0}^{\mathrm{T}}=\Omega^{x}$. We assume the target-space ghost number to be bounded from above and hence the degree to be bounded from below. Without loss of generality, this bound can again be assumed to be zero. The graded finite-dimensional space $\mathcal{H}^{\mathrm{T}}$ can be decomposed as $\mathcal{H}^{\mathrm{T}}=\mathcal{E} \oplus \mathcal{F} \oplus \mathcal{G}$ with $\operatorname{Ker} \Omega^{y} \supset \mathcal{E} \cong H\left(\Omega^{y}, \mathcal{H}^{\mathrm{T}}\right)$ and $\mathcal{G}=\operatorname{Im}\left(\Omega^{y}\right)$. Be$\mathcal{G F}$

cause the conditions of Proposition 3.6 are then satisfied, $\Omega^{\mathrm{T}}$ is invertible, and the system can be reduced to $\left(\widetilde{\Omega}^{\mathrm{T}}, \Gamma(\mathcal{E})\right)$.

Explicitly, it follows from (3.12) that the reduced BRST operator $\widetilde{\Omega}^{\mathrm{T}}$ is given by

$$
\imath \widetilde{\Omega}^{\mathrm{T}}=\boldsymbol{d}+\widetilde{\sigma}, \quad \tilde{\sigma}=\stackrel{\mathcal{E} \mathcal{G}}{\sigma}-\stackrel{\mathcal{E F}}{\sigma}\left(\stackrel{\mathcal{G} \mathcal{F}}{\sigma}+\imath \stackrel{\mathcal{G F}}{\Omega^{y}}\right)^{-1} \stackrel{\mathcal{G} \mathcal{G}}{\sigma} .
$$

Here, we have used that $\boldsymbol{d} \Gamma(\mathcal{E}) \subset \Gamma(\mathcal{E})$, and we also have used $\boldsymbol{d}$ to denote the restriction of $\boldsymbol{d}$ to $\Gamma(\mathcal{E})$. Similar conventions are used for operators that restrict to a subspace.

The inverse of $\frac{\mathcal{G F}}{\sigma}+\imath \mathcal{G F}^{y}$ involved in (4.24) is constructed as follows. If $\rho: \mathcal{G} \rightarrow \mathcal{F}$ is the inverse to $i \Omega^{y}: \mathcal{F} \rightarrow \mathcal{G}$, then

$$
\left(\stackrel{\mathcal{G F}}{\sigma}+\imath \stackrel{\mathcal{G F}}{\Omega^{y}}\right)^{-1}=\rho-\rho \stackrel{\mathcal{G F}}{\sigma} \rho+\rho \stackrel{\mathcal{G F}}{\sigma} \rho \mathcal{G \mathcal { F }} \sigma-\ldots
$$

Accordingly, $\widetilde{\sigma}: \mathcal{E} \rightarrow \mathcal{E}$ takes the form

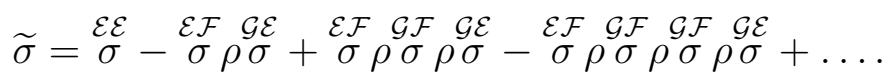


The $\ell$ th term in this expansion has target-space ghost number $1-\ell$ and form degree $\ell$. In particular, all terms after the $d$ th term vanish because there cannot be terms of form degree higher than $d$, the dimension of $X$. Furthermore, if the $\Omega^{y}$-cohomology vanishes below target-space ghost number $k_{\min }$ and above target-space ghost number $k_{\max }$, then all terms after the $\left(k_{\max }-k_{\min }+1\right)$ th term also vanish.

With $\widetilde{\Psi}^{\mathrm{T}}$ denoting the string field associated with $\mathcal{E}$, the equations of motion for the reduced system are given by

$$
(\boldsymbol{d}+\widetilde{\sigma}) \widetilde{\Psi}^{\mathrm{T}(0)}=0
$$

with gauge transformations described by

$$
\delta \widetilde{\Psi}^{\mathrm{T}(0)}=(\boldsymbol{d}+\widetilde{\sigma}) \widetilde{\Psi}^{\mathrm{T}(1)},
$$

where the ghost-number- 1 component fields of $\widetilde{\Psi}^{\mathrm{T}(1)}$ are to be interpreted as gauge parameters. We claim that equations (4.27) are the unfolded form of the original equations in the sense of [20, 21, 55].

4.4. From the parent theory to the unfolded formulation. Examples. We illustrate the general method of reducing the parent system to its unfolded form as in Sec. 4.3 with two examples, the relativistic particle and the Fronsdal system of higher-spin fields.

Klein-Gordon equations. We first consider the simplest example, with the parent system the relativistic particle described by the BRST operator $\Omega=\theta \eta^{\mu \nu} p_{\mu} p_{\nu}$, whence $\Omega^{y}=\theta \eta^{\mu \nu} p_{\mu}^{y} p_{\nu}^{y}$. The space $\mathcal{H}^{\mathrm{T}}$ can be viewed as the space of formal power series in $y^{\mu}$ and $\theta, \mathcal{C}^{\mu}$. The cohomology of $\Omega^{y}$ in $\mathcal{H}^{\mathrm{T}}$ can easily be shown to be represented by the space $\mathcal{E} \subset \mathcal{H}^{\mathrm{T}}$ of $\theta$-independent formal power series in $y^{\mu}, \mathcal{C}^{\mu}$ corresponding to traceless tensors in $y^{\mu}$, i.e., the series satisfying the constraint

$$
\eta_{\mu \nu} \frac{\partial}{\partial y^{\mu}} \frac{\partial}{\partial y^{\nu}} f(y, \mathcal{C})=0
$$

With the degree being minus the target-space ghost number (i.e., minus the homogeneity degree in $\theta$ in our case), the cohomology is concentrated in one degree and we see that $\widetilde{\sigma}={ }_{\sigma}^{\mathcal{E}}=\mathcal{C}^{\mu} \widetilde{p}_{\mu}^{y}$, where $\widetilde{p}_{\mu}^{y}$ is the action of $p_{\mu}^{y}$ in $\mathcal{E}$. Next, because there are no states in negative target-space ghost numbers, the physical fields, which are component fields of $\widetilde{\Psi}^{\mathrm{T}(0)}$, are associated with $\mathcal{C}^{\mu}$-independent states and are therefore traceless symmetric tensor fields. The equations of motion then take the form

$$
\left(\partial_{\mu}-\widetilde{p}_{\mu}^{y}\right) \widetilde{\Psi}^{\mathrm{T}(0)}=0 .
$$

These equations are the unfolded form of the Klein-Gordon equation (see [24]).

We note that in all models where the cohomology of $\Omega^{y}$ vanishes in negative ghost numbers, as is the case in this example, it follows from (4.28) that the component fields contained in $\widetilde{\Psi}^{\mathrm{T}(0)}$ are gauge invariant, because there are no gauge transformations that affect them. 
Fronsdal equations. As another example of the reduction in Sec. 4.3, we now construct the unfolded form of the Fronsdal system described in Sec. 3.5

For the BRST operator given by (3.43) and (3.33), the operator $\Omega^{y}$ is obtained by replacing $p_{\mu}$ with $p_{\mu}^{y}$ (see (4.3)). The representation space $\mathcal{H}^{\mathrm{T}}$ can be taken as the space of formal power series in the variables $y^{\mu}$ and polynomials in $a^{\dagger \mu}$ and in the ghosts $\mathcal{C}^{\mu}, \eta, \xi, c^{\dagger}, b^{\dagger}$. The operator $\imath \Omega^{y}$ acts in the representation space as

$$
\imath \Omega^{y}=-\imath \theta \square+c^{\dagger} S+S^{\dagger} \frac{\partial}{\partial b^{\dagger}}-\imath c^{\dagger} \frac{\partial}{\partial b^{\dagger}} \frac{\partial}{\partial \theta}+\imath \xi T-2 \imath \xi \frac{\partial}{\partial b^{\dagger}} \frac{\partial}{\partial c^{\dagger}},
$$

where we introduce the notation

$$
\square=\eta^{\mu \nu} \frac{\partial}{\partial y^{\mu}} \frac{\partial}{\partial y^{\nu}}, \quad S=\eta^{\mu \nu} \frac{\partial}{\partial a^{\dagger \mu}} \frac{\partial}{\partial y^{\nu}}, \quad S^{\dagger}=a^{\dagger \mu} \frac{\partial}{\partial y^{\mu}}, \quad T=\eta^{\mu \nu} \frac{\partial}{\partial a^{\dagger \mu}} \frac{\partial}{\partial a^{\dagger \nu}} .
$$

We remind the reader that the total BRST operator is

$$
\imath \Omega^{\mathrm{T}}=\boldsymbol{d}+\sigma+\imath \Omega^{y}
$$

with $\boldsymbol{d}$ and $\sigma$ defined in (4.10).

To calculate the result of the reduction described in Sec.4.3, it is convenient to proceed in two steps. At the first step, we obtain an intermediate system, whose further reduction gives the unfolded form of the Fronsdal equations.

The total BRST operator $\Omega^{\mathrm{T}}$ splits as in (3.17), with the underlying grading given by minus the homogeneity degree in $\theta, c^{\dagger}$, and $\xi$ :

$$
\begin{aligned}
\Omega^{\mathrm{T}} & =\Omega_{-1}^{\mathrm{T}}+\Omega_{0}^{\mathrm{T}}, \\
\imath \Omega_{-1}^{\mathrm{T}}=-\imath \theta \square+c^{\dagger} S+\imath \xi T, & \Omega_{0}^{\mathrm{T}}=\boldsymbol{d}+\sigma+S^{\dagger} \frac{\partial}{\partial b^{\dagger}}-\imath c^{\dagger} \frac{\partial}{\partial b^{\dagger}} \frac{\partial}{\partial \theta}-2 \imath \xi \frac{\partial}{\partial b^{\dagger}} \frac{\partial}{\partial c^{\dagger}} .
\end{aligned}
$$

The first step of the reduction is to the cohomology of $\Omega_{-1}^{\mathrm{T}}$, which we now describe.

\section{Lemma 4.3.}

(1) For $d=1,2, \quad H_{0}\left(\Omega_{-1}^{\mathrm{T}}, \mathcal{H}^{\mathrm{T}}\right) \neq 0, H_{1}\left(\Omega_{-1}^{\mathrm{T}}, \mathcal{H}^{\mathrm{T}}\right) \neq 0$, and $H_{i}\left(\Omega_{-1}^{\mathrm{T}}, \mathcal{H}^{\mathrm{T}}\right)=0$ for $i \geqslant 2$.

(2) For $d \geqslant 3, H_{0}\left(\Omega_{-1}^{\mathrm{T}}, \mathcal{H}^{\mathrm{T}}\right) \neq 0$ and $H_{i}\left(\Omega_{-1}^{\mathrm{T}}, \mathcal{H}^{\mathrm{T}}\right)=0$ for $i \geqslant 1$.

Moreover, in both cases, the space $H_{0}\left(\Omega_{-1}^{\mathrm{T}}, \mathcal{H}^{\mathrm{T}}\right)$ is isomorphic to the space $\widehat{\mathcal{E}}$ of elements $\Phi\left(y, a^{\dagger}, b^{\dagger}, \mathcal{C}\right)$ of $\mathcal{H}^{\mathrm{T}}$ satisfying the relations

$$
\square \Phi=0, \quad S \Phi=0, \quad T \Phi=0 .
$$

Proof. The cohomology is to be calculated in the space of functions that are formal power series in the variables $y^{\mu}$ and polynomials in $a^{\dagger \mu}, \mathcal{C}^{\mu}, b^{\dagger}, \theta, c^{\dagger}$, and $\xi$. The variables $\mathcal{C}^{\mu}$ and $b^{\dagger}$ and their conjugates are not involved in $\Omega_{-1}^{T}$, and hence they enter the cohomology as tensor factors. We omit this dependence in the rest of the proof. Next, $\imath \Omega_{-1}^{\mathrm{T}}$ is homogeneous in $a^{\dagger \mu}$ and $y^{\mu}$, of degree -2 . This implies that the cohomology can be calculated on 
the polynomials that have a definite total homogeneity degree in $\left(y^{\mu}, a^{\dagger \mu}\right)$. We can thus evaluate the cohomology in the space of polynomials in $y^{\mu}, a^{\dagger \mu}, \theta, c^{\dagger}$, and $\xi$.

The BRST operator consists of three pairwise commuting parts, and hence its cohomology can be evaluated as the cohomology of $c^{\dagger} S$ on the cohomology of $\xi T$ evaluated on the cohomology of $\theta \square$. The cohomology of $\xi T$ on the cohomology of $\theta \square$ is given by the space of all polynomials in $y^{\mu}, a^{\dagger \mu}$, and $c^{\dagger}$ that are annihilated by $T$ and $\square$. Indeed, because all polynomials in $y^{\mu}$ can be written as $\square$ acting on some polynomial in $y^{\mu}$, the cohomology of $\theta \square$ reduces to $\theta$-independent polynomials in $y^{\mu}$ that are in the kernel of $\square$. The same reasoning can be applied for the cohomology of $\xi T$ in the cohomology of $\theta \square$. We are therefore left with the "utmost" cohomology of $c^{\dagger} S$. Let $\mathcal{N}=\operatorname{ker} T \cap \operatorname{ker} \square$ on the space of polynomials in $y^{\mu}$ and $a^{\dagger \mu}$ and let $S_{\mathcal{N}}$ denote the restriction of $S$ to $\mathcal{N}$.

We note that whenever $S_{\mathcal{N}}$ has the property that $\operatorname{Im} S_{\mathcal{N}}=\mathcal{N}$, the cohomology is concentrated in degree zero and is given by ker $S_{\mathcal{N}} \subset \mathcal{N}$, and is therefore described by conditions (4.35). But this property of $S_{\mathcal{N}}$ is proved in Appendix $\mathbf{A}$ for $d \geqslant 3$, which shows assertion (2). The proof in the Appendix is based on the relation of the BRST operator to the $s p(4)$ algebra and involves standard representation-theory techniques that allow finding the occurrences of singular vectors in Verma and generalized Verma modules. In short, the proof amounts to showing that for $d \geqslant 3, s p(4)$ Verma modules contain no singular vectors except the one generated by a suitable power of the operator $\bar{S}^{\dagger}$. For $d=1,2$, the analysis shows that $S_{\mathcal{N}}$ has a nonzero cokernel in $\mathcal{N}$ because of the existence of additional singular vectors; finding their number and positions then yields assertion (1).

We restrict to the case $d \geqslant 3$ in what follows.

By proposition 3.6. the system $\left(\Omega^{\mathrm{T}}, \Gamma\left(\mathcal{H}^{\mathrm{T}}\right)\right)$ can be algebraically reduced to $\left(\widehat{\Omega}^{\mathrm{T}}, \Gamma(\widehat{\mathcal{E}})\right)$ with

$$
\imath \widehat{\Omega}^{\mathrm{T}}=\boldsymbol{d}+\sigma+S^{\dagger} \frac{\partial}{\partial b^{\dagger}} .
$$

Let $\widehat{\Psi}^{\mathrm{T}}$ be the string field associated with the subspace $\widehat{\mathcal{E}}$. Its ghost-number-zero component is

$$
\widehat{\Psi}^{\mathrm{T}(0)}=\widehat{\Psi}^{0}+b^{\dagger} \widehat{\Psi}^{1}, \quad \widehat{\Psi}^{1}=\mathcal{C}^{\mu} \widehat{\Psi}_{\mu}^{1}
$$

(where $\widehat{\Psi}^{0}$ and $\widehat{\Psi}_{\mu}^{1}$ are associated with the subspaces in $\widehat{\mathcal{E}}$ of the respective target-space ghost numbers 0 and -1 ). Hence, we have the following proposition.

Proposition 4.4. The parent field theory for the Fronsdal free higher-spin gauge theory can be reduced to the field theory associated with the intermediate system $\left(\widehat{\Omega}^{\mathrm{T}}, \Gamma(\widehat{\mathcal{E}})\right)$ by elimination of generalized auxiliary fields. The associated equations of motion are 
explicitly given by

$$
\begin{aligned}
& (\boldsymbol{d}+\sigma) \widehat{\Psi}^{0}=-S^{\dagger} \widehat{\Psi}^{1} \\
& (\boldsymbol{d}+\sigma) \widehat{\Psi}^{1}=0
\end{aligned}
$$

The main feature of the intermediate system $\left(\widehat{\Omega}^{T}, \Gamma(\widehat{\mathcal{E}})\right)$ is that the states (and the associated fields) are only constrained by trace conditions 4.35) and that the reduced BRST operator remains simple after the elimination of a considerable amount of states (which correspond to algebraically contractible pairs).

Having obtained the intermediate system $\left(\widehat{\Omega}^{\mathrm{T}}, \Gamma(\widehat{\mathcal{E}})\right)$, we proceed with the second step of the reduction. Following the strategy described in Sec. 4.3 , we now perform the reduction from $\left(\widehat{\Omega}^{\mathrm{T}}, \Gamma(\widehat{\mathcal{E}})\right)$ to another system $\left(\widetilde{\Omega}^{\mathrm{T}}, \Gamma(\mathcal{E})\right)$ using decomposition (3.17) again, this time with the underlying grading given by minus the target-space ghost number, which is in the case of $\widehat{\mathcal{E}}$ the homogeneity degree in $b^{\dagger}$ :

$$
\widehat{\Omega}_{-1}^{\mathrm{T}}=S^{\dagger} \frac{\partial}{\partial b^{\dagger}}, \quad \widehat{\Omega}_{0}^{\mathrm{T}}=\boldsymbol{d}+\sigma .
$$

To describe the reduced system, we must determine the cohomology of $\widehat{\Omega}_{-1}^{\mathrm{T}}$ and evaluate the reduction of the BRST operator.

We first find the cohomology of $\Omega_{-1}^{\mathrm{T}}$. We recall that $d \geqslant 3$ and also recall the notation $\bar{S}^{\dagger}=y^{\mu} \frac{\partial}{\partial a^{\dagger \mu}}$ from (A.1) for a generator of the $s \ell(2)$ algebra in (A.3.

Lemma 4.5. In degree $0, H_{0}\left(\widehat{\Omega}_{-1}^{\mathrm{T}}, \widehat{\mathcal{E}}\right)$ is isomorphic to the space $\mathcal{E}_{0}$ of elements from $\mathcal{H}^{\mathrm{T}}$ of the form $\Phi\left(y, a^{\dagger}, \mathcal{C}\right)$ satisfying the relations

$$
\square \phi=0, \quad S \phi=0, \quad T \phi=0, \quad \bar{S}^{\dagger} \phi=0
$$

and $H_{1}\left(\widehat{\Omega}_{-1}^{\mathrm{T}}, \widehat{\mathcal{E}}\right)$ is isomorphic to the space $\mathcal{E}_{1}$ of elements in $\mathcal{H}^{\mathrm{T}}$ of the form $b^{\dagger} \lambda\left(y, a^{\dagger}, \mathcal{C}\right)$ satisfying the relations

$$
\square \lambda=0, \quad S \lambda=0, \quad T \lambda=0, \quad S^{\dagger} \lambda=0 .
$$

In what follows, we use the notation $\mathcal{E}=\mathcal{E}_{0} \oplus \mathcal{E}_{1} \cong H\left(\widehat{\Omega}_{-1}^{\mathrm{T}}, \widehat{\mathcal{E}}\right)$.

We note that the elements of $\widehat{\mathcal{E}}$ specified in the proposition depend on $\mathcal{C}^{\mu}$ arbitrarily; as functions of $y^{\mu}$ and $a^{\dagger \mu}$, they decompose into monomials. Those singled out by (4.40) correspond to traceless tensors described by the Young tableaux

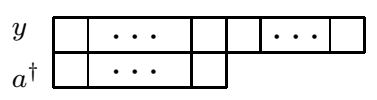

(where the length of the first row is greater than or equal to the length of the second); the monomials singled out by (4.41) correspond to traceless tensors described by the Young tableaux

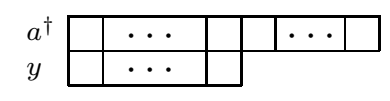


(where again the length of the first row is greater than or equal to the length of the second).

Proof of Lemma 4.5 The first three conditions in (4.40) are just the definition of the space $\widehat{\mathcal{E}}$. Generic elements of $\widehat{\mathcal{E}}$ are of the form

$$
\phi_{0}\left(y, a^{\dagger}, \mathcal{C}\right)+b^{\dagger} \phi_{1}\left(y, a^{\dagger}, \mathcal{C}\right)
$$

where $\phi_{0}$ and $\phi_{1}$ satisfy these conditions separately. We write $\widehat{\mathcal{E}}=\widehat{\mathcal{E}}_{0} \oplus \widehat{\mathcal{E}}_{1}$ accordingly.

The BRST operator $\widehat{\Omega}_{-1}^{\mathrm{T}}$ annihilates $\phi_{0}$ trivially, and to identify a unique representative in the cohomology, we must fix the freedom of changing $\phi_{0}$ by a coboundary. The space $\widehat{\mathcal{E}}_{0}$ decomposes into a direct sum of finite-dimensional representations of the $s \ell(2)$ algebra in (A.3). (We recall that the representations are finite-dimensional because for any monomial $p$, there exist two positive integer numbers $n$ and $m$ such that $\left(S^{\dagger}\right)^{n} p=0$ and $\left(\bar{S}^{\dagger}\right)^{m} p=0$.) In each irreducible summand, there exists a unique vector (the lowest-weight vector) that is not in the image of $S^{\dagger}$, and it is singled out by the condition $\bar{S}^{\dagger} \phi_{0}=0$. This gives the statement about $H_{0}$. The statement about $H_{1}$ is obvious.

The space $\widehat{\mathcal{E}}$ can be decomposed as

$$
\widehat{\mathcal{E}}=\mathcal{E}_{0} \oplus \mathcal{E}_{1} \oplus \widetilde{\mathcal{F}} \oplus \widetilde{\mathcal{G}}
$$

where $\widetilde{\mathcal{G}}=\operatorname{Im} \widehat{\Omega}_{-1}^{\mathrm{T}} \subset \widehat{\mathcal{E}}_{0}$. Furthermore, exchanging the roles of $S^{\dagger}$ and $\bar{S}^{\dagger}$ in the proof of Lemma 4.5, we can also show that $\widetilde{\mathcal{F}}$ can be taken as $\widetilde{\mathcal{F}}=b^{\dagger} \bar{S}^{\dagger} \widehat{\mathcal{E}} \subset \widehat{\mathcal{E}}_{1}$, which we do in what follows. We also note that because the operators defining the direct summands in (4.45) have definite homogeneity degrees in both $y^{\mu}$ and $a^{\dagger \mu}$, the projectors to the summands preserve the bidegree $(m, n)$ in $\left(a^{\dagger \mu}, y^{\mu}\right)$.

To complete the reduction to the unfolded formalism, in accordance with Proposition 4.2 we next calculate the reduction of the BRST operator $\widehat{\Omega}^{\mathrm{T}}$ given in (4.36) to $\mathcal{E}$.

We only need to compute the reduced differential $\widetilde{\sigma}$, see (4.24). By Lemma 4.5, the cohomology of $\Omega_{-1}^{\mathrm{T}}$ is concentrated only in degrees 0 and 1 , and therefore (see the paragraph following (4.26), $\tilde{\sigma}$ becomes

$$
\widetilde{\sigma}=\stackrel{\mathcal{E} \mathcal{E}}{\sigma}-\stackrel{\mathcal{E} \widetilde{\mathcal{F}}}{\sigma} \rho \tilde{\mathcal{G}} \mathcal{E}
$$

where $\rho: \widetilde{\mathcal{G}} \rightarrow \widetilde{\mathcal{F}}$ is the inverse of $\imath \widetilde{\mathcal{G}}_{-1}^{\widetilde{\mathcal{F}}}=S^{\dagger} \frac{\partial}{\partial b^{\dagger}}$. Moreover, the degree of $\frac{\mathcal{E} \widetilde{\mathcal{F}}}{\sigma} \rho \tilde{\mathcal{G}} \mathcal{E}$ is 1 , which implies that $\stackrel{\mathcal{E} \widetilde{\mathcal{F}}}{\sigma} \rho \tilde{\mathcal{G}} \mathcal{E}$ is nonvanishing only as a map from $\mathcal{E}^{0}$ to $\mathcal{E}^{1}$. Along with the homogeneity degree in $b^{\dagger}$, we now use the degrees in $a^{\dagger \mu}$ and $y^{\mu}$. Namely, $\widehat{\Omega}_{-1}^{\mathrm{T}}$ on $\widehat{\mathcal{E}}$ is of bidegree $(1,-1)$ in $\left(a^{\dagger}, y\right)$ and therefore its inverse must also be homogeneous, and of bidegree $(-1,1)$. Because $\sigma$ is of bidegree $(0,-1)$, it follows that $\stackrel{\mathcal{E} \widetilde{\mathcal{F}}}{\sigma} \tilde{\mathcal{G}} \tilde{\sigma}$ has bidegree $(-1,-1)$. But $\mathcal{E}_{0}$ contains monomials with the number of $y$ 's greater than or equal to the number of $a^{\dagger}$ 's, while $\mathcal{E}_{1}$ contains monomials with the number of $y$ 's less than or equal to 
the number of $a^{\dagger}$ 's. We therefore conclude that $\stackrel{\mathcal{E} \widetilde{\mathcal{F}}}{\sigma} \frac{\widetilde{\mathcal{G}}}{\sigma}$ is nonvanishing only on monomials represented by rectangular Young tableaux. These monomials belong to the intersection of the kernels of $h, S^{\dagger}$, and $\bar{S}^{\dagger}$ (and are invariants of the $s \ell(2)$ algebra in (A.3).

For a monomial $\phi \in \mathcal{E}_{0}$ corresponding to a rectangular Young tableaux,

$$
\rho \stackrel{\widetilde{\mathcal{G}} \mathcal{E}}{\sigma} \phi=b^{\dagger} \bar{S}^{\dagger} \sigma \phi
$$

Indeed, using $S^{\dagger} \bar{S}^{\dagger} \phi=0$ and $\left[S^{\dagger}, \bar{S}^{\dagger}\right] \phi=h \phi=0$, we obtain that $\bar{S}^{\dagger} S^{\dagger} \phi=0$, which implies $S^{\dagger} \phi=0$ because no polynomials in $\mathcal{E}_{0}$ can be in the image of $S^{\dagger}$. Applying then $\imath \Omega_{-1}^{\widehat{\mathcal{E}} \widehat{\mathcal{E}}}=S^{\dagger} \frac{\partial}{\partial b^{\dagger}}$ to both sides and using $\bar{S}^{\dagger} \phi=S^{\dagger} \phi=h \phi=0$, we see that Eq. (4.47) is equivalent to $\widetilde{\mathcal{G}} \sigma \phi=\sigma \phi$. (We here need the explicit choice of $\widetilde{\mathcal{F}}$ made above, which guarantees that $\imath \Omega_{-1}^{\widehat{\mathcal{E}} \widehat{\mathcal{E}}}$ is invertible). This last equation is satisfied because for $\phi \in \mathcal{E}_{0}$ corresponding to a rectangular Young tableau, $\sigma \phi=\frac{\partial}{\partial b^{\dagger}} S^{\dagger} \bar{S}^{\dagger} \sigma \phi \in \widetilde{\mathcal{G}}$.

Let $\Pi^{\mathcal{R}}$ be the projector on the subspace of polynomials in $\mathcal{E}_{0}$ corresponding to rectangular Young tableaux (see Appendix $\mathbb{B}$ for an explicit expression). Applying $\sigma$ to the right-hand side of (4.47) and defining $\bar{\sigma}=-\mathcal{C}^{\mu} \frac{\partial}{\partial a^{\dagger \mu}}$, we then see that for any $\phi \in \mathcal{E}_{0}$,

$$
\stackrel{\mathcal{E} \widetilde{\mathcal{F}}}{\sigma} \stackrel{\widetilde{\mathcal{G}} \mathcal{E}}{\rho} \phi=b^{\dagger} \sigma \bar{\sigma} \Pi^{\mathcal{R}} \phi
$$

where the right-hand side of (4.48) belongs indeed to $\mathcal{E}_{1}$ because it is annihilated by $S^{\dagger}$. Finally, the reduced BRST differential is given by

$$
\imath \widetilde{\Omega}^{\mathrm{T}} \boldsymbol{\phi}=\left[\boldsymbol{d}+\stackrel{\mathcal{E} \mathcal{E}}{\sigma}-b^{\dagger} \sigma \bar{\sigma} \Pi^{\mathcal{R}}\right] \boldsymbol{\phi}, \quad \boldsymbol{\phi} \in \Gamma(\mathcal{E}) .
$$

In order to obtain an explicit expression for $\stackrel{\mathcal{E} \mathcal{\sigma}}{\sigma}$, we need the projector $\Pi^{\mathcal{E}_{0}}$, whose explicit expression is given in Appendix $\left[\mathbf{B}\right.$ (The projector $\Pi^{\mathcal{E}_{1}}$ is not needed because $\sigma$ restricts to $\mathcal{E}_{1}$.)

We write $\widetilde{\Psi}^{\mathrm{T}(0)}$ for the ghost-number-zero part of the string field $\left(\widehat{\Psi}^{\mathrm{T}}\right)^{\mathcal{E}}$ associated with $\mathcal{E}$. Its has the form

$$
\widetilde{\Psi}^{\mathrm{T}(0)}=\widetilde{\Psi}_{0}+b^{\dagger} \widetilde{\Psi}_{1}, \quad \widetilde{\Psi}_{1}=\mathcal{C}^{\mu} \widetilde{\Psi}_{1 \mu},
$$

where $\widetilde{\Psi}^{0}$ and $b^{\dagger} \widetilde{\Psi}_{1 \mu}$ are associated with the respective spaces $\mathcal{E}_{0}$ and $\mathcal{E}_{1}$. The corresponding equations of motion are a reformulation of the unfolded form of the Fronsdal equations [56]. ${ }^{6}$

Proposition 4.6 (unfolded Fronsdal equations). The equations of motion $\widetilde{\Omega}^{\mathrm{T}} \widetilde{\Psi}^{\mathrm{T}(0)}=0$ are given by

$$
\left(\boldsymbol{d}+\Pi^{\mathcal{E}_{0}} \sigma\right) \widetilde{\Psi}_{0}=0
$$

\footnotetext{
${ }^{6}$ The authors are grateful to M. Vasiliev for drawing their attention to a problem in an earlier attempt to derive equations 4.51-4.52).
} 


$$
(\boldsymbol{d}+\sigma) \widetilde{\Psi}_{1}=-\sigma \bar{\sigma} \Pi^{\mathcal{R}} \widetilde{\Psi}_{0}
$$

Under the gauge transformations $\delta \widetilde{\Psi}^{\mathrm{T}(0)}=\widetilde{\Omega}^{\mathrm{T}} \widetilde{\Psi}^{\mathrm{T}(1)}$ with $\widetilde{\Psi}^{\mathrm{T}(1)}=-\imath b^{\dagger} \widetilde{\Lambda}$, the component fields in $\widetilde{\Psi}_{0}$ are invariant and can therefore be interpreted as generalized curvatures, while the component fields in $\widetilde{\Psi}_{1}$, to be interpreted as generalized gauge fields, transform as

$$
\delta \widetilde{\Psi}_{1}=(\boldsymbol{d}+\sigma) \widetilde{\Lambda}
$$

where the component fields in $\widetilde{\Lambda}$, which are of ghost number 1 , are to be replaced with gauge parameters. Because the component fields $\widetilde{\Psi}^{\mathrm{T}(1)}$ are associated with states at ghost number -1 , the tensor structure of the component fields of $\widetilde{\Lambda}$ and hence also of the gauge parameters is described by 4.43).

\section{DisCUSSION}

Summary. The main result in this paper can be summarized by the diagram

$$
\begin{aligned}
& \text { Parent Field Theory } \\
& \qquad\left(\boldsymbol{d}+\sigma+\imath \Omega^{y}, \Psi^{\mathrm{T}}\right)
\end{aligned}
$$
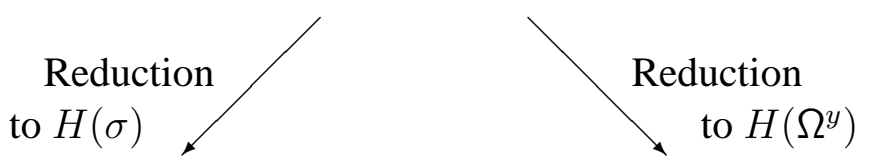

$$
\begin{aligned}
& \text { Original Field Theory } \\
& \qquad(\imath \Omega, \Psi)
\end{aligned}
$$

\section{Unfolded Formulation}

$\left(\boldsymbol{d}+\widetilde{\sigma}, \widetilde{\Psi}^{\mathrm{T}}\right)$

Curved backgrounds. At the first-quantized level, the construction of the extended firstorder system is a simplified and adapted version of Fedosov quantization, which has been developed in order to study quantization of curved manifolds. This is the reason why the construction of the extended system can naturally be done also for a quantum system on a curved background. Furthermore, using the full power of the Fedosov method provides a framework to study the extension of quantum systems from flat to curved backgrounds. We plan to discuss these matters in more details elsewhere.

Symmetries. We also comment on how global symmetries and their representations enter the parent system. The standard setting [21, 55] of the unfolded formalism consists in specifying a Lie algebra $\mathfrak{g}$ of global symmetries and its representation. The fields take values in this representation and the equations have the form of the covariant constancy condition with respect to a flat $\mathfrak{g}$-connection. These data inherently occur in the parent 
system: the representation is given by $H^{0}\left(\Omega^{y}, \mathcal{H}^{\mathrm{T}}\right)$ and the enveloping algebra of $\mathfrak{g}$ in this representation is given by $H^{0}\left(\left[\Omega^{y}, \cdot\right]\right)$.

This realization of the unfolded setting in the BRST theory could be expected for the following reasons. At the first-quantized level, elements of $H^{0}([\Omega, \cdot])$ are naturally interpreted as observables of the corresponding system. At the level of the associated field theory, these elements then determine linear global symmetries.

In the example of the Klein-Gordon equations (see Sec. 4.3), the BRST cohomology $H^{0}\left(\Omega^{y}, \mathcal{H}^{\mathrm{T}}\right)$ can be calculated and agrees with the algebra obtained in [57].

Interactions. In the Lagrangian case, the appropriate deformation theory for studying consistent interactions has been developed in [42] (see also [58]). It is based on the graded differential algebra composed of local functionals in the fields and antifields graded by ghost number, the field-antifield antibracket, and the Batalin-Vilkovisky master action. In the non-Lagrangian context, according to Sec. 3.1 the field theory is described by a BRST differential $s$ that is a nilpotent evolutionary vector field on the space of fields and their derivatives. The graded differential algebra on which deformation theory is based is then composed of the space of evolutionary vector fields in the fields and their derivatives, graded again by ghost number, the commutator bracket $[\cdot, \cdot]$ for evolutionary vector fields and the vector field $s$. As usual [59], it follows from

$$
\frac{1}{2}\left[s+g^{(1)}+\ldots, s+g^{(1)}+\ldots\right]=0,
$$

where $g$ is some deformation parameter, that first-order nontrivial interactions $\stackrel{(1)}{s}$ are representatives of the cohomology of the adjoint action of $s$ in ghost number 1, while obstructions to first-order deformations are controlled by the bracket induced in the cohomology. Because the adjoint cohomology of $s$ in the space of evolutionary vector fields is invariant under the elimination or introduction of generalized auxiliary fields, so are the nontrivial consistent interactions.

If the expansion is in terms of homogeneity in the fields, for instance

(1)

$$
\stackrel{(1)}{s}=\stackrel{(1)}{K^{A}}{ }_{B C}\left(\psi^{B}, \psi^{C}\right) \frac{\partial}{\partial \psi^{A}}+\partial_{\mu}\left(\stackrel{(1)}{K}_{B C}^{A}\left(\psi^{B}, \psi^{C}\right) \frac{\partial}{\partial \psi_{, \mu}^{A}}\right)+\ldots,
$$

with $K^{A}{ }_{B C}\left(\psi^{B}, \psi^{C}\right)$ denoting local functions of homogeneity 2 in the fields and their derivatives, the deformation problem can be entirely reformulated at the first-quantized level: the existence of a consistent deformation corresponds to the existence of multilinear graded symmetric differential operators $K: \Gamma(\mathcal{H})^{\otimes n} \rightarrow \Gamma(\mathcal{H})$ of ghost number 1 for $n \geqslant 2$ that combine with $\Omega$ into an $L_{\infty}$ algebra, see, e.g., [60].

Of course, after having found a consistent deformation of the differential $s$, additional constraints need to be satisfied in order for this deformation to be associated with a (real) Lagrangian gauge field theory. 
Acknowledgments. We are grateful to K. Alkalaev, G. Bonelli, N. Boulanger, B.L. Feigin, E.B. Feigin, O. Sheynkman, P. Sundell, and especially M.A. Vasiliev for useful discussions. MG and IYuT thank M. Henneaux for kind hospitality at Université Libre de Bruxelles. The work of GB and MG is supported in part by a "Pôle d'Attraction Interuniversitaire" (Belgium), by IISN-Belgium, convention 4.4505.86, and by the European Commission RTN program HPRN-CT00131, in which the authors are associated to K. U. Leuven. GB is also supported by Proyectos FONDECYT 1970151 and 7960001 (Chile); MG and AMS are supported in part by the INTAS (Grant 00-00262), the RFBR Grant 04-01-00303, 02-01-00930, and by the Grant LSS-1578.2003.2; IYuT is supported in part by the RFBR Grant 02-02-16944 and by the Grant LSS-1578.2003.2.

\section{ApPendix A. Structure of SOME POlynomial $s p(4)$ REPRESENTATIONS}

For the operators $\square, S$, and $T$ involved in the BRST operator $\Omega_{-1}^{\mathrm{T}}$ in (4.34), we here show a property that allows calculating the cohomology of $\Omega_{-1}^{\mathrm{T}}$ acting on the space $\mathcal{P}_{d}\left(y, a^{\dagger}\right)$ of polynomials in $y^{\mu}$ and $a^{\dagger \mu}, \mu=1, \ldots, d$ (tensored with the appropriate ghosts). This can be done by standard representation-theory methods.

The space $\mathcal{P}_{d}\left(y, a^{\dagger}\right)$ carries representations of two Lie algebras, the Lorentz algebra acting in the standard way and the $\operatorname{sp}(4)$ algebra whose Chevalley basis generators are represented as

$$
T=\eta^{\mu \nu} \frac{\partial}{\partial a^{\dagger \mu}} \frac{\partial}{\partial a^{\dagger \nu}}, \quad S^{\dagger}=a^{\dagger \mu} \frac{\partial}{\partial y^{\mu}}, \quad S=\eta^{\mu \nu} \frac{\partial}{\partial a^{\dagger \mu}} \frac{\partial}{\partial y^{\nu}}, \quad \square=\eta^{\mu \nu} \frac{\partial}{\partial y^{\mu}} \frac{\partial}{\partial y^{\nu}},
$$

$$
\begin{array}{ll}
h^{\prime}=-a^{\dagger \nu} \frac{\partial}{\partial a^{\dagger \nu}}-\frac{d}{2}, & h=a^{\dagger \mu} \frac{\partial}{\partial a^{\dagger \mu}}-y^{\mu} \frac{\partial}{\partial y^{\mu}}, \\
\bar{T}=-\frac{1}{4} \eta^{\mu \nu} a_{\mu}^{\dagger} a_{\nu}^{\dagger}, \quad \bar{S}^{\dagger}=y^{\mu} \frac{\partial}{\partial a^{\dagger \mu}}, \quad \bar{S}=y^{\mu} a_{\mu}^{\dagger}, \quad \bar{\square}=y^{\mu} y^{\nu} \eta_{\mu \nu} .
\end{array}
$$

These two algebras commute, and by Howe duality [61, 62], $\mathcal{P}_{d}\left(y, a^{\dagger}\right)$ decomposes into a direct sum of irreducible $s p(4)$ representations. We can therefore restrict the analysis to an individual irreducible $s p(4)$ representation.

With the space $\mathfrak{h}^{*}$ dual to the Caratan subalgebra of $\operatorname{sp}(4)$ identified with the standard plane $\mathbb{R}^{2}$, the $s p(4)$ root diagram can be represented as

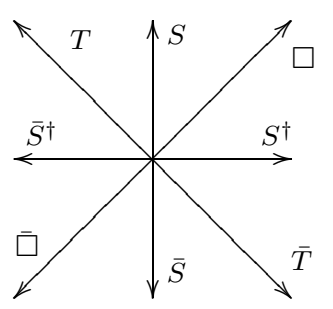

There is the "horizontal" $s \ell(2)$ algebra generated by $e=S^{\dagger}, f=\bar{S}^{\dagger}$, and $h$, with the commutation relations given by

$$
[e, f]=h, \quad[h, e]=2 e, \quad[h, f]=-2 f .
$$


Each irreducible $\operatorname{sp}(4)$ representation $\mathcal{J}_{\lambda}$ occurring in the decomposition of $\mathcal{P}_{d}\left(y, a^{\dagger}\right)$ is a highest-weight representation, with the highest-weight vector defined by the annihilation conditions $S^{\dagger} v_{\lambda}=T v_{\lambda}=0$ (which clearly imply $\square v_{\lambda}=0=T v_{\lambda}$ ). The condition $S^{\dagger} v_{\lambda}=0$ also implies that the corresponding polynomial in $\left(a^{\dagger \mu}, y^{\mu}\right)$ has the symmetry of the Young tableaux ${ }_{y}^{a^{\dagger}}$\begin{tabular}{ll|l|l|l|} 
& $\cdots$ & $\cdots$ & $\cdots$ \\
\hline
\end{tabular} . In particular, if the polynomial is of bidegree $(m, n)$ in $\left(a^{\dagger \mu}, y^{\mu}\right)$, it follows that $m \geqslant n \geqslant 0$. We also let $\mathcal{K}_{\lambda}$ denote the representation of the horizontal $s \ell(2)$ subalgebra generated from $v_{\lambda}$. It is finite-dimensional because any monomial $p$ satisfies the conditions $\left(S^{\dagger}\right)^{n} p=0$ and $\left(\bar{S}^{\dagger}\right)^{m} p=0$ for two positive integers $n$ and $m$.

Each $\mathcal{J}_{\lambda}$ is in fact a quotient of (or coincides with) the generalized Verma module $\mathcal{W}_{\lambda}$ induced from a finite-dimensional representation $\mathcal{K}_{\lambda}$. A generalized Verma module can be simply defined in our setting as the space of polynomials in $\bar{\square}, \bar{S}$, and $\bar{T}$ with coefficients in $\mathcal{K}_{\lambda} \cdot{ }^{7}$ Therefore, instead of the highest-weight vector in an ordinary Verma module, there is a representation of the horizontal $s \ell(2)$ algebra in a generalized Verma module.

We next follow the standard analysis [63] that consists in studying the shifted Weyl group action and finding the weights in the Weyl group orbit that are in the fundamental Weyl chamber. To describe the representation weights, we introduce elements $\varepsilon_{1}$ and $\varepsilon_{2}$ of the orthonormal basis in $\mathfrak{h}^{*}$. In the above root diagram, $\varepsilon_{1}$ and $\varepsilon_{2}$ are identified as the roots corresponding to $S$ and $S^{\dagger}$ respectively. The two positive simple roots $\alpha$ and $\alpha^{\prime}$ are given by

$$
\alpha=\varepsilon_{2}, \quad \alpha^{\prime}=\varepsilon_{1}-\varepsilon_{2},
$$

and hence are the roots corresponding to $S^{\dagger}$ and $T$ respectively. Half the sum of positive roots is given by $\rho=\frac{3}{2} \varepsilon_{1}+\frac{1}{2} \varepsilon_{2}$. The weights are parameterized as

$$
\begin{gathered}
\lambda=a \varepsilon_{1}+b \varepsilon_{2}, \\
a=-\frac{1}{2}(m+n+d), \quad b=\frac{1}{2}(m-n),
\end{gathered}
$$

where we recall that $m \geqslant n \geqslant 0$.

The Weyl group action shifted by $-\rho$ is generated by the two mappings

$$
(a, b) \mapsto(a,-b-1) \quad \text { and } \quad(a, b) \mapsto(b-1, a+1)
$$

The fundamental Weyl chamber $\mathcal{C}_{-\rho}$ (translated by $-\rho$ ) is determined by the conditions

$$
a \varepsilon_{1}+b \varepsilon_{2} \in \mathcal{C}_{-\rho} \Longleftrightarrow\left(b+\frac{1}{2} \geqslant 0, \quad a-b+1 \geqslant 0\right) .
$$

These conditions are to be examined for each element of the Weyl group orbit of A.5, with $m \geqslant n \geqslant 0$. With the dominant weight found, the Bruhat order on the Weyl group induces an order on the weights. For generalized Verma modules, the analysis differs

\footnotetext{
${ }^{7}$ More formally, let $\mathfrak{p}$ be the parabolic subalgebra in $s p(4)$ generated by $\bar{S}^{\dagger}, T, S^{\dagger}$, and $h^{\prime}$ and let $\mathcal{K}_{\lambda}$ be a representation of $\mathfrak{p}$ where $T, S$, and $\square$ act trivially. Then $\mathcal{W}_{\lambda}=\mathcal{U}(s p(4)) \otimes \mathcal{u}(\mathfrak{p}) \mathcal{K}_{\lambda}$.
} 
from the standard in that only those weights are kept in the orbit whose component "along the horizontal subalgebra" (the $\varepsilon_{2}$-component in our case) is dominant (see, e.g., [64] for details in the case of generalized Verma modules).

The Bruhat order induced on the representation weights depends on the dimension $d$, and analysis shows that for $d=1,2$, some of the representations in the decomposition are quotients of the corresponding generalized Verma modules over nontrivial submodules, but for $d \geqslant 3$, they necessarily coincide with the generalized Verma modules.

For $d \geqslant 3$, this has the following implication for the properties of the $\square, S$, and $T$ operators. As in the proof of Lemma 4.3 let $\mathcal{N}$ be the subspace of polynomials annihilated by $T$ and $\square$ in a given irreducible representation $\mathcal{J}_{\lambda}$ and $S_{\mathcal{N}}$ be the restriction of $S$ to $\mathcal{N}$. Then $\operatorname{Im} S_{\mathcal{N}}=\mathcal{N}$.

Indeed, in an irreducible representation $\mathcal{J}_{\lambda}$, let $\mathcal{J}_{\lambda}^{\ell}$ denote the subspace spanned by elements of the form $\square^{\ell_{1}} \bar{T}^{\ell_{2}} \bar{S}^{\ell_{3}} \chi$, where $\chi \in \mathcal{K}_{\lambda}$ and $\ell_{1}+\ell_{2}+\ell_{3}=\ell$ with $\ell, \ell_{i} \geqslant 0$. The property $\operatorname{Im} S_{\mathcal{N}}=\mathcal{N}$ is equivalently reformulated as the property that all subspaces $\mathcal{N}_{\lambda}^{\ell}=\operatorname{ker} T \cap \operatorname{ker} \square \cap J_{\lambda}^{\ell}$ are isomorphic for $\ell \geqslant 0$. On the other hand, we can expand $\phi^{\ell} \in \mathcal{J}_{\lambda}^{\ell}$ into the $T$ - and $\square$-traceless part and the respective traces as

$$
\phi^{\ell}=\phi_{0,0}^{\ell}+\sum_{\ell^{\prime}=1}^{\ell} \sum_{\substack{\ell_{1}, \ell_{2} \geqslant 0 \\ \ell_{1}+\ell_{2}=\ell}} \bar{\square}^{\ell_{1}} \bar{T}^{\ell_{1}} \phi_{\ell_{1}, \ell_{2}}^{\ell},
$$

where $\phi_{\ell_{1}, \ell_{2}}^{\ell} \in \mathcal{N}^{\ell-\ell_{1}-\ell_{2}}$. This immediately gives the formula

$$
\operatorname{dim} \mathcal{J}_{\lambda}^{\ell}=\sum_{\ell^{\prime}<\ell}\left(\ell^{\prime}+1\right) \operatorname{dim} \mathcal{N}_{\lambda}^{\ell-\ell^{\prime}} .
$$

But as we have shown, $\mathcal{J}_{\lambda}=\mathcal{W}_{\lambda}$. An elementary calculation of the character of the generalized Verma module then shows that

$$
\operatorname{dim} \mathcal{J}_{\lambda}^{\ell}=\operatorname{dim} \mathcal{W}_{\lambda}^{\ell}=\left(\operatorname{dim} \mathcal{K}_{\lambda}\right) \frac{(\ell+1)(\ell+2)}{2} .
$$

Comparing A.9) and (A.10), we conclude that $\operatorname{dim} \mathcal{N}_{\lambda}^{\ell}=\operatorname{dim} \mathcal{K}_{\lambda}$, independently of $\ell$, and therefore the $\mathcal{N}_{\lambda}^{\ell}$ spaces are isomorphic for all $\ell$. As noted above, this is equivalent to the desired property of $S_{\mathcal{N}}$.

\section{Remarks.}

(i) The BRST operator $\Omega_{-1}^{\mathrm{T}}$ in (4.34) evaluates the cohomology of the Abelian subalgebra in $s p(4)$ generated by $T, S$, and $\square$. The occurrence of generalized Verma modules may be traced to the fact that the annihilation conditions with respect to these three operators constitute the highest-weight conditions in the $s p(4)$ generalized Verma modules. The "opposite" operators $\bar{T}, \bar{S}$, and $\bar{\square}$ are then the creation operators in the generalized Verma modules. 
(ii) In the language of the standard $s p(4)$ Verma modules, the statement about the structure of $\mathcal{J}_{\lambda}$ means that for $d \geqslant 3$, each irreducible representation $\mathcal{J}_{\lambda}$ is a quotient of the Verma module $\mathcal{V}_{\lambda}$ with respect to only one singular vector, given by $\left(\bar{S}^{\dagger}\right)^{2 b+1} v_{\lambda}$, but for $d=1,2$, some of the representations $\mathcal{J}_{\lambda}$ are given by a quotient of $\mathcal{V}_{\lambda}$ over $t$ wo singular vectors.

\section{APPENDiX B. PROJECTORS}

The horizontal $s \ell(2)$ algebra in A.3, which played a crucial role in the proof of Lemma 4.5, is also helpful in writing the projectors $\Pi^{\mathcal{R}}$ and $\Pi^{\mathcal{E}_{0}}$ that occur in (4.48)(4.52). We first consider the projector

$$
\Pi^{\mathcal{E}_{0}}: \widehat{\mathcal{E}}_{0} \rightarrow \mathcal{E}_{0},
$$

where $\widehat{\mathcal{E}}_{0}$ is a direct sum of finite-dimensional irreducible $s \ell(2)$ representations and $\mathcal{E}_{0}$, defined in Lemma 4.5 is the subspace of lowest-weight vectors (those annihilated by the $f$ generator of $s \ell(2))$. We have [65]

$$
\Pi^{\mathcal{E}_{0}}=\sum_{n \geqslant 0} \frac{1}{n !} \frac{1}{\prod_{j=1}^{n}(h-j-1)} e^{n} f^{n}=\prod_{n \geqslant 1}\left(1+\frac{e f}{n(h-n-1)}\right) .
$$

It is easy to verify that $f \Pi^{\mathcal{E}_{0}}=\Pi^{\mathcal{E}_{0}} e=0$ : for example, for $f \Pi^{\mathcal{E}_{0}}$, the relevant commutation relations are

$$
\left[f, e^{n}\right]=-n(h-n+1) e^{n-1}
$$

and

$$
f F(h)=F(h+2) f
$$

for "any" function $F$. Therefore, taking the $n$th term in (B.2), we have

$$
\begin{gathered}
f \cdot \frac{1}{n !} \frac{1}{\prod_{j=1}^{n}(h-j-1)} e^{n} f^{n} \\
=\frac{1}{n !} \frac{1}{\prod_{j=1}^{n}(h-j+1)}\left(-n(h-n+1) e^{n-1} f^{n}+e^{n} f^{n+1}\right) \\
=-\frac{1}{(n-1) !} \frac{1}{\prod_{j=1}^{n-1}(h-j+1)} e^{n-1} f^{n}+\frac{1}{n !} \frac{1}{\prod_{j=1}^{n}(h-j+1)} e^{n} f^{n+1},
\end{gathered}
$$

which gives zero after summation over $n$.

Next, the operator $\Pi^{\mathcal{R}}$ occurred in Lemma 4.5 as the projector on rectangular two-row Young tableaux. In terms of the $s \ell(2)$ algebra, it is the projector on $s \ell(2)$ invariants (the 
trivial representation) and can therefore be written as

$$
\Pi^{\mathcal{R}}=\prod_{n \geqslant 1}\left(1-\frac{C}{n(n+2)}\right)=-\frac{2 \sin (\pi \sqrt{1+C})}{\pi C \sqrt{1+C}},
$$

where $C=4 e f+h^{2}-2 h$ is the $s \ell(2)$ Casimir operator. This projector simplifies on the image of $\Pi^{\mathcal{E}_{0}}$ :

$$
\Pi^{\mathcal{R}} \Pi^{\mathcal{E}_{0}}=\frac{2 \sin (\pi h)}{\pi h(h+1)(h+2)} \Pi^{\mathcal{E}_{0}}
$$

\section{REFERENCES}

[1] M. Fierz and W. Pauli, "On relativistic wave equations for particles of arbitrary spin in an electromagnetic field," Proc. Roy. Soc. Lond. A173 (1939) 211-232.

[2] L. P. S. Singh and C. R. Hagen, "Lagrangian formulation for arbitrary spin. 1. The boson case," Phys. Rev. D9 (1974) 898-909.

[3] L. P. S. Singh and C. R. Hagen, "Lagrangian formulation for arbitrary spin. 2. The fermion case," Phys. Rev. D9 (1974) 910-920.

[4] C. Fronsdal, "Massless fields with integer spin," Phys. Rev. D18 (1978) 3624.

[5] J. Fang and C. Fronsdal, "Massless fields with half integral spin," Phys. Rev. D18 (1978) 3630.

[6] S. Ouvry and J. Stern, "Gauge fields of any spin and symmetry," Phys. Lett. B177 (1986) 335.

[7] A. K. H. Bengtsson, "A unified action for higher spin gauge bosons from covariant string theory," Phys. Lett. B182 (1986) 321.

[8] M. Henneaux and C. Teitelboim, First and second quantized point particles of any spin, ch. 9, pp. 113-152. Quantum mechanics of fundamental systems 2, Centro de Estudios Científicos de Santiago. Plenum Press, 1987.

[9] A. K. H. Bengtsson, "BRST approach to interacting higher spin gauge fields," Class. Quant. Grav. 5 (1988) 437.

[10] L. Cappiello, M. Knecht, S. Ouvry, and J. Stern, "BRST construction of interacting gauge theories of higher spin fields," Ann. Phys. 193 (1989) 10.

[11] A. K. H. Bengtsson, "BRST quantization in anti-de Sitter space and gauge fields," Nucl. Phys. B333 (1990) 407.

[12] J. M. F. Labastida, "Massless particles in arbitrary representations of the Lorentz group," Nucl. Phys. B322 (1989) 185.

[13] A. Pashnev and M. Tsulaia, "Description of the higher massless irreducible integer spins in the BRST approach," Mod. Phys. Lett. A13 (1998) 1853, hep-th/9803207

[14] G. Bonelli, "On the tensionless limit of bosonic strings, infinite symmetries and higher spins," Nucl. Phys. B669 (2003) 159-172, hep-th/0305155

[15] G. Bonelli, "On the covariant quantization of tensionless bosonic strings in AdS spacetime," JHEP 11 (2003) 028, hep-th/0309222

[16] A. Sagnotti and M. Tsulaia, "On higher spins and the tensionless limit of string theory," Nucl. Phys. B682 (2004) 83-116, hep-th/0311257.

[17] X. Bekaert, I. L. Buchbinder, A. Pashnev, and M. Tsulaia, "On higher spin theory: Strings, BRST, dimensional reductions," Class. Quant. Grav. 21 (2004) S1457-1464, hep-th/ 0312252

[18] M. A. Vasiliev, "Equations of motion of interacting massless fields of all spins as a free differential algebra," Phys. Lett. B209 (1988) 491-497. 
[19] M. A. Vasiliev, "Consistent equations for interacting massless fields of all spins in the first order in curvatures," Annals Phys. 190 (1989) 59-106.

[20] M. A. Vasiliev, "Unfolded representation for relativistic equations in (2+1) anti-De Sitter space," Class. Quant. Grav. 11 (1994) 649-664.

[21] M. A. Vasiliev, "Higher spin gauge theories: Star-product and AdS space," hep-th/9910096

[22] M. A. Vasiliev, "Conformal higher spin symmetries of 4D massless supermultiplets and osp(L,2M) invariant equations in generalized (super)space," Phys. Rev. D66 (2002) 066006, hep-th/0106149

[23] M. A. Vasiliev, "Higher spin gauge theories in various dimensions," Fortsch. Phys. 52 (2004) 702-717, hep-th/0401177

[24] O. V. Shaynkman and M. A. Vasiliev, "Scalar field in any dimension from the higher spin gauge theory perspective," Theor. Math. Phys. 123 (2000) 683-700, hep-th/0003123

[25] E. S. Fradkin and G. A. Vilkovisky, "Quantization of relativistic systems with constraints," Phys. Lett. B55 (1975) 224.

[26] I. A. Batalin and G. A. Vilkovisky, "Relativistic S matrix of dynamical systems with boson and fermion constraints," Phys. Lett. B69 (1977) 309-312.

[27] E. S. Fradkin and T. E. Fradkina, "Quantization of relativistic systems with boson and fermion first and second class constraints," Phys. Lett. B72 (1978) 343.

[28] M. Henneaux, "Hamiltonian form of the path integral for theories with a gauge freedom," Phys. Rept. 126 (1985) 1.

[29] T. W. B. Kibble, "Geometrization of quantum mechanics," Commun. Math. Phys. 65 (1979) 189.

[30] A. Heslot, "Quantum mechanics as a classical theory," Phys. Rev. D 31 (1985) 1341-1348.

[31] B. Hatfield, "Quantum field theory of point particles and strings,". Redwood City, USA: Addison-Wesley (1992) 734 p. (Frontiers in physics, 75).

[32] T. Schilling, Geometry of quantum mechanics. PhD thesis, The Pennsylvania State University, 1996.

[33] A. Ashtekar and T. A. Schilling, "Geometrical formulation of quantum mechanics," gr-qc/9706069

[34] G. Barnich and M. Grigoriev, "Hamiltonian BRST and Batalin-Vilkovisky formalisms for second quantization of gauge theories," Commun. Math. Phys. 254 (2005) 581-601, hep-th/0310083

[35] M. R. Gaberdiel and B. Zwiebach, "Tensor constructions of open string theories I: Foundations," Nucl. Phys. B505 (1997) 569-624, hep-th/9705038

[36] P. Olver, Applications of Lie Groups to Differential Equations. Spinger Verlag, New York, 2nd ed., 1993. 1st ed., 1986.

[37] I. Anderson, “The variational bicomplex" tech. rep., Formal Geometry and Mathematical Physics, Department of Mathematics, Utah State University, 1989.

[38] C. B. Thorn, "Perturbation theory for quantized string fields," Nucl. Phys. B287 (1987) 61.

[39] M. Bochicchio, "Gauge fixing for the field theory of the bosonic string," Phys. Lett. B193 (1987) 31.

[40] M. Bochicchio, "String field theory in the Siegel gauge," Phys. Lett. B188 (1987) 330.

[41] C. B. Thorn, "String field theory," Phys. Rept. 175 (1989) 1-101.

[42] G. Barnich and M. Henneaux, "Consistent couplings between fields with a gauge freedom and deformations of the master equation," Phys. Lett. B311 (1993) 123-129, hep-th/9304057

[43] G. Barnich, F. Brandt, and M. Henneaux, "Local BRST cohomology in the antifield formalism. I. General theorems," Commun. Math. Phys. 174 (1995) 57-92, hep-th/9405109

[44] G. Barnich, F. Brandt, and M. Henneaux, "Local BRST cohomology in gauge theories," Phys. Rept. 338 (2000) 439-569, hep-th/ 0002245 
[45] A. Dresse, P. Grégoire, and M. Henneaux, "Path integral equivalence between the extended and nonextended Hamiltonian formalisms," Phys. Lett. B245 (1990) 192.

[46] M. Henneaux and C. Teitelboim, Quantization of Gauge Systems. Princeton University Press, 1992.

[47] I. Anderson, "Introduction to the variational bicomplex," in Mathematical Aspects of Classical Field Theory, M. Gotay, J. Marsden, and V. Moncrief, eds., vol. 132 of Contemporary Mathematics, pp. 51-73. Amer. Math. Soc., 1992.

[48] A. K. H. Bengtsson, "An abstract interface to higher spin gauge field theory," hep-th/0403267

[49] B. Zwiebach, "Closed string field theory: Quantum action and the B-V master equation," Nucl. Phys. B390 (1993) 33-152, hep-th/9206084.

[50] I. A. Batalin and E. S. Fradkin, "Operatorial quantization of dynamical systems subject to second class constraints," Nucl. Phys. B279 (1987) 514.

[51] I. A. Batalin, E. S. Fradkin, and T. E. Fradkina, "Generalized canonical quantization of dynamical systems with constraints and curved phase space," Nucl. Phys. B332 (1990) 723.

[52] B. Fedosov, "Deformation quantization and index theory,". Berlin, Germany: Akademie-Verl. (1996) 325 p. (Mathematical topics: 9).

[53] M. A. Grigoriev and S. L. Lyakhovich, "Fedosov deformation quantization as a BRST theory," Commun. Math. Phys. 218 (2001) 437-457, hep-th/ 0003114

[54] I. A. Batalin, M. A. Grigoriev, and S. L. Lyakhovich, "Star product for second class constraint systems from a BRST theory," Theor. Math. Phys. 128 (2001) 1109-1139, hep-th/0101089

[55] O. V. Shaynkman, I. Y. Tipunin, and M. A. Vasiliev, "Unfolded form of conformal equations in M dimensions and o(M+2)-modules," hep-th/0401086

[56] V. E. Lopatin and M. A. Vasiliev, "Free massless bosonic fields of arbitrary spin in d- dimensional de Sitter space," Mod. Phys. Lett. A3 (1988) 257.

[57] M. G. Eastwood, "Higher symmetries of the Laplacian," hep-th/0206233

[58] M. Henneaux, "Consistent interactions between gauge fields: The cohomological approach," in Secondary Calculus and Cohomological Physics, A. V. M. Henneaux, J. Krasil'shchik, ed., vol. 219 of Contemporary Mathematics, pp. 93-109. Amercian Mathematical Society, 1997. hep-th/9712226

[59] M. Gerstenhaber, "On the deformation of rings and algebras," Ann. of Math. 79 (1964) 59-103.

[60] T. Lada and J. Stasheff, "Introduction to SH Lie algebras for physicists," Int. J. Theor. Phys. 32 (1993) 1087-1104, hep-th/9209099

[61] R. Howe, "Transcending classical invariant theory," J. Amer. Math. Soc. 3 (1989) 2.

[62] R. Howe, "Remarks on classical invariant theory," Trans. Amer. Math. Soc. 2 (1989) 313.

[63] J. Dixmier, Algèbres enveloppantes. Gauthier-Villars, Paris, 1974.

[64] V. Mazorchuk, Generalized Verma Modules. VNTL-Klasyka Publishers, 1999.

[65] D. Zhelobenko, Representations of the reductive Lie algebras. Nauka, Moscow, 1994. 